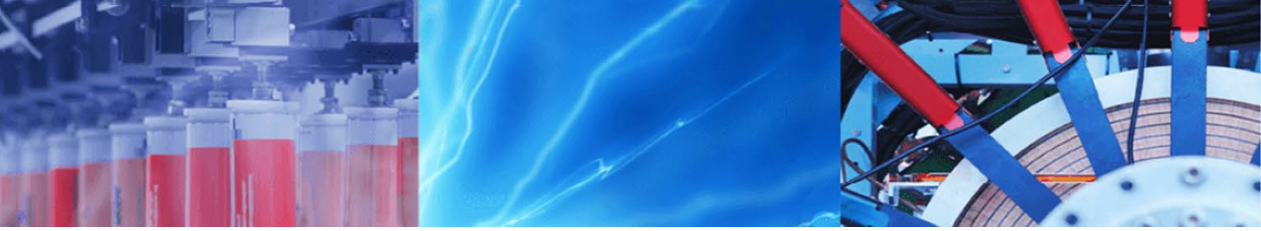

Research Article

\title{
Nonlinear vibrations of single- and double-walled carbon nanotubes resting on two-parameter foundation in a magneto-thermal environment
}

\author{
M. G. Sobamowo ${ }^{1}$ (D) J. O. Akanmu² - O. A. Adeleye ${ }^{3} \cdot$ A. A. Yinusa ${ }^{1}$
}

() Springer Nature Switzerland AG 2019

\begin{abstract}
The excellent mechanical, electrical, structural and thermal properties coupled with high strength to weight ratio of carbon nanotubes have tremendously expanded their applications in various industrial, engineering, physical and natural sciences processes. In this work, nonlocal elasticity theory is used to analyze nonlinear vibrations of single and doublewalled carbon nanotubes resting on two-parameter foundation in a thermal and magnetic environment. With the aid of Galerkin decomposition method, the systems of nonlinear partial differential equations are transformed into systems of nonlinear ordinary differential equations which are solved using homotopy perturbation method. The developed analytical solutions are used to investigate the influences of elastic foundations, magnetic field, temperature rise, interlayer forces, small scale parameter and boundary conditions on the frequency ratio. From the results, it is observed that the frequency ratio for all boundary conditions decreases as the number of walls increases from single to double. Also, it is established that the frequency ratio is highest for clamped-simple supported and lowest for clamped-clamped supported. Additionally, the results revealed that the frequency ratio decreases with increase in the value of spring constant $\left(k_{1}\right)$ temperature and magnetic field strength. This work will enhance the applications of carbon nanotubes in structural, electrical, mechanical and biological applications especially in a thermal and magnetic environment.
\end{abstract}

Keywords Single and double-walled carbon nanotubes · Magneto-thermal environment $\cdot$ Nonlocal elastic theory . Small-scale effects · Elastic foundations

\section{Introduction}

The novel nanostructure materials discovered by lijima [1] have led to considerable number of studies on carbon nanotubes due to their promising applications in nanodevices, nanoelectronics, and nanocomposites. Also, the excellent mechanical, electrical, structural and thermal properties coupled with high strength to weight ratio property of carbon nanotubes have continuously and tremendously expanded their applications in various industrial, engineering, physical and natural sciences processes. In fact, the nanostructures have merits when applied to the functionability of transistors and diodes. However, carbon nanotubes (CNTs) are capable of undergoing large deformations within the elastic limit and vibrate at frequency in the order of $\mathrm{GHz}$ and $\mathrm{THz}$. Consequently, logical investigations and analysis of carbon nanotube have been a subject of interest such as the vibrations of a micro-resonator that is excited by electrostatic and piezoelectric actuations. Various studies have been carried out on beams, carbon nanotube, nano-wires, nano-rods and nano-beam so as to specifically understand and achieve their area of best fit [2-13]. In achieving this, the well know beam models were employed and dynamic

$\triangle$ M. G. Sobamowo, mikegbeminiyi@gmail.com; mikegbeminiyiprof@yahoo.com|'Department of Mechanical Engineering, University of Lagos, Akoka, Lagos, Nigeria. ${ }^{2}$ Department of Civil and Environmental Engineering, University of Lagos, Akoka, Lagos, Nigeria. ${ }^{3}$ Department of System Engineering, University of Lagos, Akoka, Lagos, Nigeria. 
ranges were obtained in the scope of the structures. In such studies, Liew et al. [5], Pantano et al. [6, 7], Qian et al. [8] and Salvetat et al. [9] examined the mechanics of single and multiwalled carbon nanotubes. Sears and Batra [10] analyzed carbon nanotubes buckling under the influence of axial compression. Yoon et al. [11] and Wang and Cai [12] investigated the impacts of initial stress on multiwall carbon nanotube with a focus on non-coaxial resonance. Wang et al. [13] explored the dynamic response of multiwalled carbon nanotubes using Timoshenko beam model. Zhang et al. [14] scrutinized the influence of compressive axial load on the transverse dynamic behaviour of doublewalled carbon nanotubes (DWCNT). Another work on the vibration of double-walled carbon nanotubes was presented by Elishakoff and Pentaras [15]. Also, studies on nonlinear vibration of nanomechanical resonator, nanotube and nanowire-based electromechanical systems have been carried out by Buks and Yurke [16] and Postma et al. [17] while Fu et al. [18] examined nonlinear vibration analysis of embedded carbon nanotubes. In the same year, Xu et al. [19] considered the dynamic response of a double-walled carbon nanotube under the influence of nonlinear intertube van der Waals forces. The vibration of carbon nanotube-based switches with focus on static and dynamic responses was analyzed by Dequesnes et al. [20]. Few years later, Ouakad and Younis [21] investigated the nonlinear vibration of electrically actuated carbon nanotube resonators. In an earlier work, Zamanian et al. [22] presented the non-linear vibrations analysis of a microresonator subjected to piezoelectric and electrostatic actuations. As a continuation of the tremendous work, Abdel-Rahman, Hawwa, Hajnayeb, and Belhadj [23-26] performed a vibration and instability studies of DWCNT using a nonlinear model and considering an electrostatic actuation as an external excitation agent. In their work, a DWCNT was situated and conditioned to a direct and alternating voltage and different behaviors of the nanotubes were recorded as the exciting agent is varied. They went further to determine the bifurcation point of the DWCNT and concluded that both walls have the same frequency of vibration under the two resonant conditions considered. Belhadj et al. [26] carried out the vibration analysis of a pinned-pinned supported SWCNT employing nonlocal theory of elasticity and obtained natural frequency up to third mode. The authors also put forward an explanation on the advantages of the high frequency obtained in their work to optical applications. Lei et al. [27] studied the dynamic behaviour of DWCNT by employing the well-known Timoshenko theory of beam. The nonlinear governing equations generated by Sharabiani and Yazdi [28] derived relations in the application to nanobeams that are graded and have surface roughness. Wang [29] generated a close form model for the aforementioned surface roughness effect for an unforced fluid conveying nanotubes and beams based on nonlocal theory of elasticity and ascertained the significance of the study for reasonably small thickness of the tube considered. Interesting foundation studies have been considered after modelling of CNTs as structures resting or embedded on elastic foundations such as Winkler, Pasternak and Visco-Pasternak medium [30-35]. Other interesting works through modelling and experiment have also been presented to justify the widespread application of SWCNTs [36-41].

The dynamic behaviour of SWCNTs and DWCNTs have been characterized and their dynamic behaviour have been investigated with the aids of experimental measurements, density functional theory, molecular dynamics simulations, and continuum mechanics. However, there are difficulties in performing experiment at the nanoscale level. Consequently, over the years, the classical continuum models (which do not consider the small-scale effects) have been widely applied to the small-scale structures as reviewed in the preceding section. The demerit of such classical continuum theories is witnessed in their scalefree models as they cannot incorporate the small-scale effects in their formulations. For the purpose of correcting the inadequacy in the classical continuum models, Eringen [42-45] developed nonlocal continuum mechanics based on nonlocal elasticity theory. The nonlocal elasticity theory considers the stress state at a given point to be a function of the strain field at all points in the body. Therefore, in this work, nonlocal elasticity theory is used to analyzed nonlinear vibrations of single- and double-walled carbon nanotubes resting on two-parameter foundation in a thermal and magnetic environment. With the aid of van der Waals interlayer interaction, the nested slender double-walled nanotubes are coupled with each other. Such study on the simultaneous influences of thermal and magnetic field, two-parameter foundation on the vibration of single- and double-walled carbon nanotubes using nonlocal elasticity theory has not been presented in literature. Additionally, the development of analytical expressions for the frequencies, frequency ratio and deflections of the double-walled carbon nanotubes is shown to be another novel idea of the present study. The analytical solutions are used to investigate the influences of elastic foundations, magnetic field, temperature rise, interlayer forces, small scale parameter and boundary conditions on the frequency ratio.

\section{Problem description and the governing equations}

In order to develop the governing equations of motion for the SWCNTs and DWCNTs, we first consider a SWCNT under the influence of stretching effects and resting on 
Winkler and Pasternak foundations in a thermal and magnetic environment as depicted in Fig. 1. With the aid of the Eringen's nonlocal elasticity theory, Euler-Bernoulli beam theory and Hamilton's principle, the governing equation of motion for the SWCNT is given by

$$
\begin{gathered}
E l \frac{\partial^{4} w}{\partial x^{4}}+m_{c} \frac{\partial^{2} w}{\partial t^{2}}+k_{1} w+k_{3} w^{3}-\left(\frac{E A}{2 L} \int_{0}^{L}\left(\frac{\partial w}{\partial x}\right)^{2} d x\right) \frac{\partial^{2} w}{\partial x^{2}} \\
-E A \alpha_{x} T \frac{\partial^{2} w}{\partial x^{2}}-\eta A H_{x}^{2} \frac{\partial^{2} w}{\partial x^{2}}+\left(e_{o} a\right)^{2}\left(m_{c} \frac{\partial^{4} w}{\partial x^{2} \partial t^{2}}+k_{1} \frac{\partial^{2} w}{\partial x^{2}}\right. \\
+6 k_{3} w\left(\frac{\partial w}{\partial x}\right)^{2}+3 k_{3} w^{2}\left(\frac{\partial w}{\partial x}\right)-\left(\frac{E A}{2 L} \int_{0}^{L}\left(\frac{\partial w}{\partial x}\right)^{2} d x\right) \frac{\partial^{4} w}{\partial x^{4}} \\
\left.-E A \alpha_{x} T \frac{\partial^{4} w}{\partial x^{4}}-\eta A H_{x}^{2} \frac{\partial^{4} w}{\partial x^{4}}\right)=0
\end{gathered}
$$

where $w(x, t)$ is the bending deflection of the tube, $\mathrm{t}$ is the time coordinate, $E l$ is the bending rigidity, $m_{c}$ is the mass of tube per unit length. The term $E A \alpha_{x} T$ denotes the constant axial force due to thermal effects and the term $\eta A H_{x}^{2}$ is the magnetic force per unit length due to Lorentz force exerted on the tube in z-direction. Also, A is the cross-sectional area of the tube, $\alpha_{x}$ is the coefficient of thermal expansion and $T$ is the change in temperature. Also, the term $\eta$ is the magnetic field permeability and $H_{x}$ is the magnetic field strength (Fig. 2).
For the purpose of incorporating the interlayer interactions for the DWCNTs with two layers, it is established that the pressure at any point between any two adjacent tubes depends on the difference in their deflections at that point. Therefore, one can express the linearized form of the van der Waals forces as

$F_{i}=c_{i}\left(w_{i}-w_{i-1}\right)$.

where $F_{i}$ is the van der Waals force between the ith tube and the $i-1$ th tube, $c_{i}$ is the coefficient of the van der Waals force between the $i$ th tube and the $(i-1)$ th tube. Assuming that the nested individual tubes of the DWCNT vibrate in the same plane, using the van der Waals forces in Eq. (2), the developed nonlinear governing equations of vibration for the embedded DWCNT in a thermal and magnetic environment with two layers are given as

$$
\begin{aligned}
E I_{1} & \frac{\partial^{4} w_{1}}{\partial x^{4}}+m_{c 1} 1 \frac{\partial^{2} w_{1}}{\partial t^{2}}-\left(\frac{E A_{1}}{2 L} \int_{0}^{L}\left(\frac{\partial w_{1}}{\partial x}\right)^{2} d x\right) \frac{\partial^{2} w_{1}}{\partial x^{2}} \\
& -E A_{1} \alpha_{x} T \frac{\partial^{2} w_{1}}{\partial x^{2}}-\eta A_{1} H_{x}^{2} \frac{\partial^{2} w_{1}}{\partial x^{2}}-\left(e_{0} a\right)^{2}\left(m_{c 1} \frac{\partial^{4} w_{1}}{\partial x^{2} \partial t^{2}}\right. \\
& -\left(\frac{E A_{1}}{2 L} \int_{0}^{L}\left(\frac{\partial w_{1}}{\partial x}\right)^{2} d x\right) \frac{\partial^{4} w_{1}}{\partial x^{4}}-E_{1} A \alpha_{x} T \frac{\partial^{4} w_{1}}{\partial x^{4}} \\
& \left.-\eta_{1} A H_{x}^{2} \frac{\partial^{4} w_{1}}{\partial x^{4}}+c_{1}\left(\frac{\partial^{2} w_{2}}{\partial x^{2}}-\frac{\partial^{2} w_{1}}{\partial x^{2}}\right)\right)=c_{1}\left(w_{1}-w_{2}\right)
\end{aligned}
$$

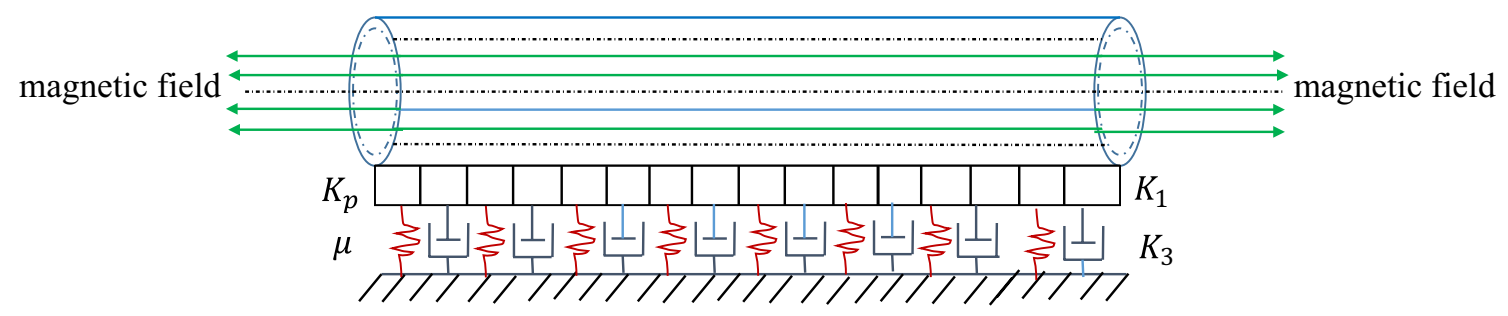

Fig. 1 The SWCNT on two-parameter elastic foundation in a thermal and magnetic field influnce

Fig. 2 The embedded DWCNT in a thermal and magnetic environment

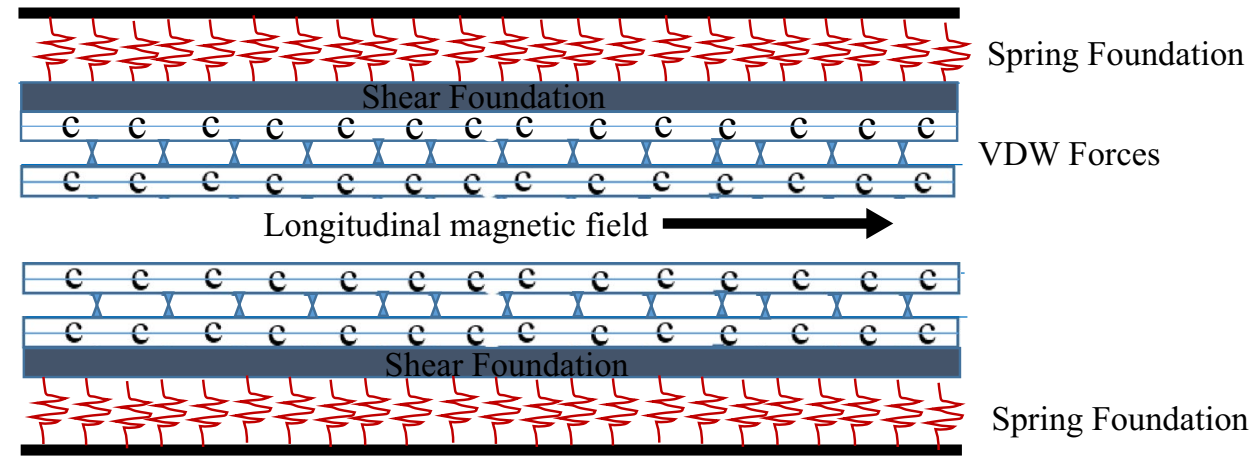

SN Applied Sciences A SPRINGER NATURE journal 


$$
\begin{aligned}
E I_{2} & \frac{\partial^{4} w_{N}}{\partial x^{4}}+m_{c 2} \frac{\partial^{2} w_{2}}{\partial t^{2}}+k_{1} w_{2}+k_{3} w_{2}^{3} \\
& -\left(\frac{E A_{2}}{2 L} \int_{0}^{L}\left(\frac{\partial w_{2}}{\partial x}\right)^{2} d x\right) \frac{\partial^{2} w_{2}}{\partial x^{2}}-E A_{2} \alpha_{x} T \frac{\partial^{2} w_{2}}{\partial x^{2}} \\
& -\eta A_{2} H_{x}^{2} \frac{\partial^{2} w_{2}}{\partial x^{2}}-\left(e_{o} a\right)^{2}\left(m_{c 2} \frac{\partial^{4} w_{2}}{\partial x^{2} \partial t^{2}}\right. \\
& +k_{1} \frac{\partial^{2} w_{2}}{\partial x^{2}}+6 k_{3} w_{2}\left(\frac{\partial w_{2}}{\partial x}\right)^{2}+3 k_{3} w_{2}^{2}\left(\frac{\partial w_{2}}{\partial x}\right) \\
& -\left(\frac{E A_{2}}{2 L} \int_{0}^{L}\left(\frac{\partial w_{2}}{\partial x}\right)^{2} d x\right) \frac{\partial^{4} w_{2}}{\partial x^{4}}-E_{2} A \alpha_{x} T \frac{\partial^{4} w_{2}}{\partial x^{4}} \\
& \left.-\eta A_{2} H_{x}^{2} \frac{\partial^{4} w_{2}}{\partial x^{4}}+c_{1}\left(\frac{\partial^{2} w_{2}}{\partial x^{2}}-\frac{\partial^{2} w_{1}}{\partial x^{2}}\right)\right)=c_{1}\left(w_{2}-w_{1}\right)
\end{aligned}
$$

It should be noted that the $k_{1}$ and $k_{3}$ will not enter into the equations of the inner tubes since only the outer tube interacts with the elastic medium.

The displacements of the nanotubes are subjected to the following boundary conditions:

For simply supported (S-S) nanotube,

$w_{i}(0, t)=0, \quad \frac{\partial^{2} w_{i}(0, t)}{\partial^{2} x}=0, \quad w_{i}(L, t)=0, \quad \frac{\left.\partial^{2} w_{i}(L, t)\right)}{\partial^{2} x}=0$.

For clamped-clamped supported (C-C) nanotube,

$w_{i}(0, t)=0, \quad \frac{\partial w_{i}(0, t)}{\partial x}=0, \quad w_{i}\left((L, t)=0, \quad \frac{\partial w_{i}(L, t)}{\partial x}=0\right.$.

For a clamped-simply supported (C-S) nanotube,

$w_{i}(0, t)=0, \quad \frac{\partial w_{i}(0, t)}{\partial x}=0, \quad w_{i}(L, t)=0, \quad \frac{\partial^{2} w_{i}((L, t)}{\partial^{2} x}=0$.

\section{Solution methodology}

Using the Galerkin's decomposition procedure to separate the spatial and temporal parts of the lateral displacement functions,

$w_{i}(x, t)=\phi(x) W_{i}(t) \quad i=1,2$.

where $w_{i}(x, t)$ is the lateral displacement functions, $W_{i}(t)$ is the time-dependent parameter or time-dependent maximum amplitude of oscillation of the $i$-th layer of the nanotube and $\phi(x)$ is a trial/comparison function that will satisfy both the geometric and natural boundary conditions.

Applying one-parameter Galerkin to a generalized form of the Eqs. (3) and (4), we have

$\int_{0}^{L} R(x, t) \phi(x) d x=0$

where $R_{N}(x, t)$ is the equation of motion for each wall. For the outer wall of double-walled carbon nanotubes,

$$
\begin{aligned}
R(x, t)= & E I_{2} \frac{\partial^{4} w_{2}}{\partial x^{4}}+m_{c 2} \frac{\partial^{2} w_{2}}{\partial t^{2}}+k_{1} w_{2} \\
& +k_{3} w_{2}^{3}-\left(\frac{E A_{2}}{2 L} \int_{0}^{L}\left(\frac{\partial w_{2}}{\partial x}\right)^{2} d x\right) \frac{\partial^{2} w_{2}}{\partial x^{2}} \\
& -E A_{2} \alpha_{x} T \frac{\partial^{2} w_{2}}{\partial x^{2}}-\eta A_{2} H_{x}^{2} \frac{\partial^{2} w_{2}}{\partial x^{2}} \\
& -\left(e_{o} a\right)^{2}\left(m_{c 2} \frac{\partial^{4} w_{2}}{\partial x^{2} \partial t^{2}}+k_{1} \frac{\partial^{2} w_{2}}{\partial x^{2}}\right. \\
& +6 k_{3} w_{2}\left(\frac{\partial w_{2}}{\partial x}\right)^{2}+3 k_{3} w_{2}^{2}\left(\frac{\partial w_{2}}{\partial x}\right) \\
& -\left(\frac{E A_{2}}{2 L} \int \frac{L}{0}\left(\frac{\partial w_{2}}{\partial x}\right)^{2} d x\right) \frac{\partial^{4} w_{2}}{\partial x^{4}} \\
& -E_{2} A \alpha_{x} T \frac{\partial^{4} w_{2}}{\partial x^{4}}-\eta A_{2} H_{x}^{2} \frac{\partial^{4} w_{2}}{\partial x^{4}} \\
& \left.+c_{1}\left(\frac{\partial^{2} w_{2}}{\partial x^{2}}-\frac{\partial^{2} w_{1}}{\partial x^{2}}\right)\right)=c_{1}\left(w_{2}-w_{1}\right)
\end{aligned}
$$

One arrives at

$$
\begin{aligned}
& \alpha_{1} E I_{2} W_{2}+\alpha_{2} m_{C 2} \frac{d^{2} W_{2}}{d t^{2}}+\alpha_{2} k_{1} W_{2}+\alpha_{3} k_{3} W_{2}^{3} \\
& -\alpha_{4} \frac{E A_{2}}{2 L} W_{N}^{3}-\left(a_{0} a\right)^{2} \alpha_{5} m_{c 2} \frac{d^{2} W_{2}}{d t^{2}} \\
& -\left(a_{0} a\right)^{2} \alpha_{5} k_{1} W_{2}-6 \alpha_{6}\left(a_{0} a\right)^{2} k_{3} W_{2}^{3} \\
& -3 \alpha_{7}\left(a_{0} a\right)^{2} k_{3} W_{2}^{3}+\alpha_{8}\left(a_{0} a\right)^{2} \frac{E A_{2}}{2 L} W_{2}^{3} \\
& +\alpha_{1}\left(a_{0} a\right)^{2}\left(E A_{2} \alpha_{x} T_{t}+\eta A_{2} H_{x}^{2}\right) W_{2} \\
& -\alpha_{5}\left(a_{0} a\right)^{2} c_{1}\left(W_{2}-W_{1}\right)-\alpha_{5}\left(E A_{2} \alpha_{x} T_{t}\right. \\
& \left.+\eta A_{2} H_{x}^{2}\right) W_{2}+\alpha_{2}\left(a_{0} a\right)^{2} c_{1}\left(W_{2}-W_{1}\right)=0
\end{aligned}
$$


After collecting like terms, we have

$$
\begin{aligned}
& \frac{d^{2} W_{2}}{d t^{2}}+\left(\frac{\alpha_{1} E I_{1}+\alpha_{2} k_{1}-\alpha_{5} \mu k_{1}+\left(\alpha_{1} \mu-\alpha_{5}\right)\left(E A_{2} \alpha_{x} T_{t}+\eta A_{2} H_{x}^{2}\right)}{\left(\alpha_{2}-\alpha_{5} \mu\right) \rho A_{2}}\right) W_{2} \\
& +\left(\frac{\left(\alpha_{2}-\alpha_{5} \mu\right) c_{1}}{\left(\alpha_{2}-\alpha_{5} \mu\right) \rho A_{2}}\right)\left(W_{2}-W_{1}\right) \\
& +\left(\frac{\alpha_{3} k_{3}-\alpha_{4} \frac{E A_{2}}{2 L}-6 \alpha_{6} \mu k_{3}-3 \alpha_{7} \mu k_{3}+\alpha_{8} \mu \frac{E A_{2}}{2 L}}{\left(\alpha_{2}-\alpha_{5} \mu\right) \rho A_{2}}\right) W_{2}^{3}=0
\end{aligned}
$$

where

$$
\begin{aligned}
& \alpha_{1}=\int_{0}^{L} \phi(x) \frac{d^{4} \phi(x)}{d x^{4}} d x, \quad \alpha_{2}=\int_{0}^{L} \phi^{2}(x) d x, \quad \alpha_{3}=\int_{0}^{L} \phi^{4}(x) d x, \\
& \alpha_{4}=\int_{0}^{L}\left(\phi(x)\left(\int_{0}^{L}\left(\frac{d \phi(x)}{\partial x}\right)^{2} d x\right) \frac{d^{2} \phi(x)}{d x^{2}} d x\right), \\
& \alpha_{5}=\int_{0}^{L} \phi(x) \frac{d^{2} \phi(x)}{d x^{2}} d x, \quad \alpha_{6}=\int_{0}^{L} \phi^{2}(x)\left(\frac{d \phi(x)}{d x}\right)^{2} d x \\
& \left.\alpha_{7}=\int_{0}^{L} \phi^{3}(x) \frac{d \phi(x)}{d x} d x, \quad \alpha_{8}=\int_{0}^{L}\left(\int_{0}^{L}\left(\frac{d \phi(x)}{\partial x}\right)^{2} d x\right) \frac{d^{4} \phi(x)}{d x^{4}} d x\right), \\
& \mu=\left(e_{0} a\right)^{2}, \quad m_{c N}=\rho A_{N},
\end{aligned}
$$

Similarly, the same procedure is applied to other inner walls appropriately.

Therefore, the governing equations of motion for nonlinear vibrations of embedded DWCNTs in a thermal and magnetic environment in ODE form is obtained as,

$$
\begin{gathered}
\frac{d^{2} W_{1}}{d t^{2}}+\left(\frac{\alpha_{1} E I_{1}+\left(\alpha_{1} \mu-\alpha_{5}\right)\left(E A_{1} \alpha_{x} T_{t}+\eta A_{1} H_{x}^{2}\right)}{\left(\alpha_{2}-\alpha_{5} \mu\right) \rho A_{1}}\right) W_{1} \\
-\frac{c_{1}}{\rho A_{1}}\left(W_{2}-W_{1}\right)+\left(\frac{\left(\alpha_{8} \mu-\alpha_{4}\right) \frac{E A_{1}}{2 L}}{\left(\alpha_{2}-\alpha_{5} \mu\right) \rho A_{1}}\right) W_{1}^{3}=0 \\
\frac{d^{2} W_{2}}{d t^{2}}+\left(\frac{\alpha_{1} E I_{2}+\left(\alpha_{1} \mu-\alpha_{5}\right)\left(E A_{2} \alpha_{x} T_{t}+\eta A_{2} H_{x}^{2}\right)}{\left(\alpha_{2}-\alpha_{5} \mu\right) \rho A_{2}}\right) W_{2} \\
+\frac{c_{1}}{\rho A_{2}}\left(W_{2}-W_{1}\right)+\left(\frac{\left(\alpha_{8} \mu-\alpha_{4}\right) \frac{E A_{2}}{2 L}}{\left(\alpha_{2}-\alpha_{5} \mu\right) \rho A_{2}}\right) W_{2}^{3}=0
\end{gathered}
$$

and the initial conditions are

$$
\begin{array}{lll}
W_{1}(0)=X & \text { and } & \frac{d W_{1}(0)}{d t}=0 \\
W_{2}(0)=X & \text { and } & \frac{d W_{2}(0)}{d t}=0
\end{array}
$$

\subsection{Homotopy perturbation method}

The nonlinear terms in Eqs. (14) and (15) make the development of exact analytical solution. Therefore, for the purpose of generating a symbolic solution for the nonlinear equations, we made a recourse homotopy perturbation method. The principle and the procedures of the method can be found in our previous works $[46,47]$.

\subsubsection{Analysis of single-walled carbon nanotube}

For SWCNT, the governing equation is given by

$$
\begin{gathered}
\frac{d^{2} W}{d t^{2}}+\left(\frac{\alpha_{1} E I+\alpha_{2} k_{1}-\alpha_{5} \mu k_{1}+\left(\alpha_{1} \mu-\alpha_{5}\right)\left(E A \alpha_{x} T_{t}+\eta A H_{x}^{2}\right)}{\left(\alpha_{2}-\alpha_{5} \mu\right) \rho A_{N}}\right) W \\
+\left(\frac{\alpha_{3} k_{3}-\alpha_{4} \frac{E A}{2 L}-6 \alpha_{6} \mu k_{3}-3 \alpha_{7} \mu k_{3}+\alpha_{8} \mu \frac{E A}{2 L}}{\left(\alpha_{2}-\alpha_{5} \mu\right) \rho A}\right) W^{3}=0
\end{gathered}
$$


Introducing the following dimensionless quantities,

$r=\sqrt{\frac{1}{A}}, \quad \tau=\omega_{0} t, \quad a=\frac{W}{r}$

After applying the dimensionless parameters in Eq. (18), Eq. (17) is transformed to

$\omega_{0}^{2} \frac{d^{2} a}{d \tau^{2}}+f_{1} a+f_{2} a^{3}=0$

where $f_{1}$ and $f_{2}$ are defined as

$f_{1}=\frac{\alpha_{1} E l+\alpha_{2} k_{1}-\alpha_{5} \mu k_{1}+\left(\alpha_{1} \mu-\alpha_{5}\right)\left(E A \alpha_{x} T_{t}+\eta A H_{x}^{2}\right)}{\left(\alpha_{2}-\alpha_{5} \mu\right) \rho A}=\omega^{2}$

$f_{2}=\left(\frac{\alpha_{3} k_{3}-\alpha_{4} \frac{E A}{2 L}-6 \alpha_{6} \mu k_{3}-3 \alpha_{7} \mu k_{3}+\alpha_{8} \mu \frac{E A}{2 L}}{\left(\alpha_{2}-\alpha_{5} \mu\right) \rho A}\right) \cdot \frac{l}{A}$

and the initial conditions are

$a(0)=X \quad$ and $\quad \frac{d a(0)}{d \tau}=0$

It is shown from Eq. (19) that

$\omega=\sqrt{f_{1}}=\sqrt{\frac{\alpha_{1} E l+\alpha_{2} k_{1}-\alpha_{5} \mu k_{1}+\left(\alpha_{1} \mu-\alpha_{5}\right)\left(E A \alpha_{x} T_{t}+\eta A H_{x}^{2}\right)}{\left(\alpha_{2}-\alpha_{5} \mu\right) \rho A}}$

It should be noted that $\omega$ is the linear forced vibration frequency, and $\omega_{0}$ is an unknown nonlinear angular frequency to be determined.

In order to solve Eq. (17), we construct the following homotopy with $\omega_{0}$ as the initial approximation for the angular nonlinear frequency as

$(1-p)\left\{\omega_{0}^{2}\left(\frac{d^{2} a}{d \tau^{2}}+a\right)\right\}+p\left(\omega_{0}^{2} \frac{d^{2} a}{d \tau^{2}}+f_{1} a+f_{2} a^{3}\right)=0$

It should be noted that the solutions of $a=a(\tau, p)$ and $\omega=\omega(p)$ of the homotopy change from their initial approximations $a_{0}=a(\tau)$ and $\omega_{0}$ to the required solutions $a(\tau)$ and $\omega_{o}$ of (19) as the embedding parameter $p$ travels from 0 to 1.

Assuming that the solution of Eq. (19) take the form of:

$a(\tau)=a_{0}(\tau)+p a_{1}(\tau)+p^{2} a_{2}(\tau)+p^{3} a_{3}(\tau)+\cdots$,

$\omega_{0}=\omega_{0}+p \omega_{1}+p^{2} \omega_{2}+p^{3} \omega_{3}+\cdots$,

After substituting Eq. (25) into the homotopy Eq. (24) and rearranging the coefficients of the terms with identical powers of $p$, we have a series of linear differential equations of the form

$p^{0}: \omega_{0}^{2}\left(\frac{d^{2} a_{0}}{d \tau^{2}}+a_{0}\right)=0$ with initial conditions

$$
\begin{aligned}
& a_{0}(0)=X \quad \text { and } \quad \frac{d a_{0}(0)}{d \tau}=0, \\
& p^{1}: \omega_{0}^{2}\left[\frac{d^{2} a_{1}}{d \tau^{2}}+a_{1}-\left(\frac{d^{2} a_{0}}{d \tau^{2}}+a_{0}\right)\right] \\
& +\omega_{0}^{2} \frac{d^{2} a_{0}}{d \tau^{2}}+f_{1} a_{0}+f_{2} a_{0}^{3}=0
\end{aligned}
$$

the corresponding initial conditions are

$$
\begin{aligned}
& a_{1}(0)=0 \text { and } \frac{d a_{1}(0)}{d \tau}=0, \\
& p^{2}: \omega_{0}^{2}\left(\frac{d^{2} a_{2}}{d \tau^{2}}+a_{2}\right)-\omega_{0}^{2} a_{1} \\
& \quad+2 \omega_{0} \omega_{1} \frac{d^{2} a_{0}}{d \tau^{2}}+f_{1} a_{1}-f_{2} a_{0}^{2} a_{1}=0
\end{aligned}
$$

And the initial conditions are given as

$a_{2}(0)=0$ and $\frac{d a_{0}(0)}{d \tau}=0$,

Since $\frac{d^{2} a_{0}}{d \tau^{2}}+a_{0}=0$ and $\frac{d^{2} a_{0}(0)}{d \tau^{2}}=-a_{0}$ from Eq. (26a), we can write Eq. (26b) as

$\omega_{0}^{2}\left(\frac{d^{2} a_{1}}{d \tau^{2}}+a_{1}\right)-\omega_{0}^{2} a_{0}+f_{1} a_{0}+f_{2} a_{0}^{3}=0$,

The solution of the initial zeroth approximation is given by

$a_{0}=X \cos \tau$,

On substituting Eq. (28) into the first approximation equation in Eq. (27), one arrives at

$\omega_{0}^{2}\left(\frac{d^{2} a_{1}}{d \tau^{2}}+a_{1}\right)-\omega_{0}^{2} X \cos \tau+f_{1} X \cos \tau+f_{2}(X \cos \tau)^{3}=0$,

After the application of trigonometry identities to the fourth-term in the LHS of Eq. (29), we have

$$
\begin{gathered}
\omega_{0}^{2}\left(\frac{d^{2} a_{1}}{d \tau^{2}}+a_{1}\right)-\omega_{0}^{2} X \cos \tau+f_{1} X \cos \tau \\
+\frac{3}{4} f_{2} X^{3} \cos \tau+\frac{1}{4} f_{2} X^{3} \cos 3 \tau=0,
\end{gathered}
$$

in order to eliminate the secular terms, we set the coefficient of $\cos \tau$ in Eq. (30) to zero

$-\omega_{0}^{2} X \cos \tau+f_{1} X \cos \tau+\frac{3}{4} f_{2} X^{3} \cos \tau=0$,

It gives the nonlinear natural frequency as,

$\omega_{0}=\sqrt{f_{1}+\frac{3}{4} f_{2} X^{2}}$ 
It should be noted that the frequency ratio is given as $\psi=\frac{\omega_{o}}{\omega}$.

Therefore, from Eqs. (32) and (23), we have the frequency ratio as

$\psi=\frac{\sqrt{f_{1}+\frac{3}{4} f_{2} X^{2}}}{\sqrt{f_{1}}}=\sqrt{1+\frac{3}{4} \frac{f_{2}}{f_{1}} X^{2}}$

On substituting Eqs. (20) and (21) into Eq. (33), we have $w(x, t)=\phi(x) a(\tau) \sqrt{\frac{l}{A}}$,

Substituting Eq. (36) and the shape functions in the Table 1 into Eq. (38), for simple simply support, we have

$w(x, t)=\left[\left(X \cos \tau+\frac{f_{2} X^{3}}{32 f_{1}+24 f_{2} X^{2}}(\cos 3 \tau-\cos \tau)\right)\right] \sqrt{\frac{l}{A}} \sin \left(\frac{n \pi x}{l}\right)$

while for clamped-clamped gives

$\psi=\sqrt{1+\frac{3}{4}\left\{\frac{\left(\alpha_{3} k_{3}-\alpha_{4} \frac{E A}{2 L}-6 \alpha_{6} \mu k_{3}-3 \alpha_{7} \mu k_{3}+\alpha_{8} \mu \frac{E A}{2 L}\right) \cdot \frac{1}{A}}{\alpha_{1} E l+\alpha_{2} k_{1}-\alpha_{5} \mu k_{1}+\left(\alpha_{1} \mu-\alpha_{5}\right)\left(E A \alpha_{x} T_{t}+\eta A H_{x}^{2}\right)}\right\} X^{2}}$

The solution of Eq. (30) produces

$a_{1}(\tau)=\frac{f_{2} X^{3}}{32 f_{1}+24 f_{2} X^{2}}(\cos 3 \tau-\cos \tau)$

Hence, the first approximate solution of Eq. (19) can be written as

$a(\tau)=a_{0}(\tau)+a_{1}(\tau)=X \cos \tau+\frac{f_{2} X^{3}}{32 f_{1}+24 f_{2} X^{2}}(\cos 3 \tau-\cos \tau)$

From Eqs. (8) and (18), we have

$w(x, t)=\phi(x) \mathrm{W}(t), \quad W=a(\tau) \sqrt{\frac{l}{A}}$

Therefore, the displacement of the nanotube to be expressed as

$$
\begin{aligned}
w(x, t)= & {\left[X \cos \tau+\frac{f_{2} X^{3}}{32 f_{1}+24 f_{2} X^{2}}(\cos 3 \tau-\cos \tau)\right] } \\
& \sqrt{\frac{I}{A}}\left\{\left(\cosh \left(\frac{\beta x}{L}\right)-\cos \left(\frac{\beta X}{L}\right)\right)\right. \\
& \left.-\left(\frac{\sinh \beta+\sin \beta}{\cosh \beta-\cos \beta}\right)\left(\sinh \left(\frac{\beta x}{L}\right)-\sin \left(\frac{\beta x}{L}\right)\right)\right\}
\end{aligned}
$$

and for clamped-simply support, one obtains

$$
\begin{aligned}
w(x, t)= & {\left[X \cos \tau+\frac{f_{2} X^{3}}{32 f_{1}+24 f_{2} X^{2}}(\cos 3 \tau-\cos \tau)\right] } \\
& \sqrt{\frac{I}{A}}\left\{\left(\cosh \left(\frac{\beta x}{L}\right)-\cos \left(\frac{\beta x}{L}\right)\right)\right. \\
& \left.-\left(\frac{\cosh \beta-\cos \beta}{\sinh \beta-\sin \beta}\right)\left(\sinh \left(\frac{\beta x}{L}\right)-\sin \left(\frac{\beta x}{L}\right)\right)\right\}
\end{aligned}
$$

\subsubsection{Analysis of double-walled carbon nanotube}

For a DWCNT, the governing equation is given by

Table 1 The basic functions corresponding to the above boundary conditions [37]

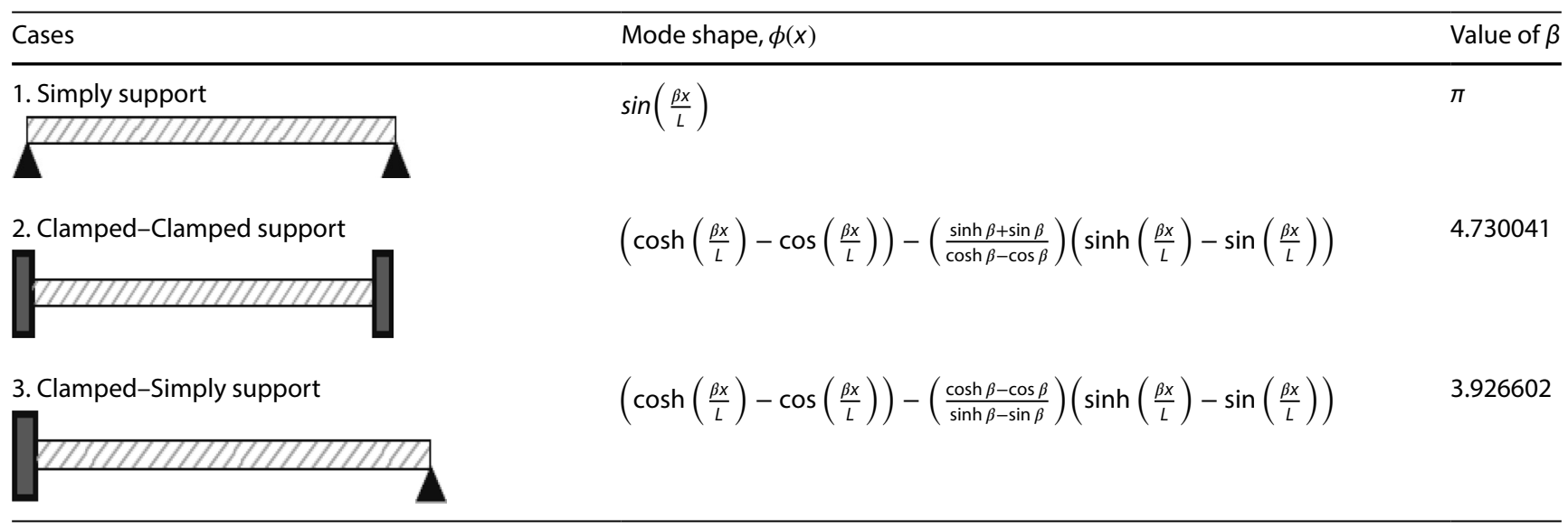




$$
\begin{aligned}
& \frac{d^{2} W_{1}}{d t^{2}}+\left(\frac{\alpha_{1} E I_{1}+\left(\alpha_{1} \mu-\alpha_{5}\right)\left(E A_{1} \alpha_{x} T_{t}+\eta A_{1} H_{x}^{2}\right)}{\left(\alpha_{2}-\alpha_{5} \mu\right) \rho A_{1}}\right) W_{1} \\
& -\frac{c_{1}}{\rho A_{1}}\left(W_{2}-W_{1}\right)+\left(\frac{\left(\alpha_{8} \mu-\alpha_{4}\right) \frac{E A_{1}}{2 L}}{\left(\alpha_{2}-\alpha_{5} \mu\right) \rho A_{1}}\right) W_{1}^{3}=0 \\
& (1-p)\left\{\omega_{0}^{2}\left(\frac{d^{2} a_{1}}{d \tau^{2}}+a_{1}\right)\right\} \\
& +p\left\{\omega_{0}^{2} \frac{d^{2} a_{1}}{d \tau^{2}}+f_{1} a_{1}+f_{2} a_{1}^{3}-f_{3} a_{2}\right\}=0
\end{aligned}
$$

In a similar manner to SWCNT, we construct a homotopy

$$
\begin{aligned}
& \frac{d^{2} W_{2}}{d t^{2}}+\left(\frac{\alpha_{1} E I_{2}+\alpha_{2} k_{1}-\alpha_{5} \mu k_{1}+\left(\alpha_{1} \mu-\alpha_{5}\right)\left(E A_{2} \alpha_{x} T_{t}+\eta A_{2} H_{x}^{2}\right)}{\left(\alpha_{2}-\alpha_{5} \mu\right) \rho A_{2}}\right) W_{2} \\
& +\frac{c_{1}}{\rho A_{2}}\left(W_{2}-W_{1}\right)+\left(\frac{\alpha_{3} k_{3}-\alpha_{4} \frac{E A_{2}}{2 L}-6 \alpha_{6} \mu k_{3}-3 \alpha_{7} \mu k_{3}+\alpha_{8} \mu \frac{E A_{2}}{2 L}}{\left(\alpha_{2}-\alpha_{5} \mu\right) \rho A_{2}}\right) W_{2}^{3}=0
\end{aligned}
$$

Using the following dimensionless parameters,

$r=\sqrt{\frac{l_{1}}{A_{1}}}, \quad a_{1}=\frac{W_{1}}{r}, \quad a_{2}=\frac{W_{2}}{r} \quad$ and $\quad \tau=\omega_{0} t$

On substituting the dimensionless parameters in Eq. (44) into Eqs. (42) and (43), we have the following dimensionless nonlinear system of equations

$\omega_{0}^{2} \frac{d^{2} a_{1}}{d \tau^{2}}+f_{1} a_{1}+f_{2} a_{1}^{3}-f_{3} a_{2}=0$,

$\omega_{0}^{2} \frac{d^{2} a_{2}}{d \tau^{2}}+g_{1} a_{2}+g_{2} a_{2}^{3}-g_{3} a_{1}=0$.

where

$$
\begin{aligned}
& f_{1}=\frac{\alpha_{1} E I_{1}+\left(\alpha_{1} \mu-\alpha_{5}\right)\left(E A_{1} \alpha_{x} T_{t}+\eta A_{1} H_{x}^{2}\right)}{\left(\alpha_{2}-\alpha_{5} \mu\right) \rho A_{1}}+\frac{c_{1}}{\rho A_{1}} \\
& f_{2}=\frac{\left(\alpha_{8} \mu-\alpha_{4}\right) E I_{1}}{2 L\left(\alpha_{2}-\alpha_{5} \mu\right) \rho A_{1}}, \quad f_{3}=\frac{c_{1}}{\rho A_{1}} \\
& g_{1}=\left(\frac{\alpha_{1} E I_{2}+\alpha_{2} k_{1}-\alpha_{5} \mu k_{1}+\left(\alpha_{1} \mu-\alpha_{5}\right)\left(E A_{1} \alpha_{x} T_{t}+\eta A_{1} H_{x}^{2}\right)}{\left(\alpha_{2}-\alpha_{5} \mu\right) \rho A_{2}}\right)+\frac{c_{1}}{\rho A_{2}} \\
& g_{2}=\frac{\left(\alpha_{3} k_{3}-\alpha_{4} \frac{E A_{2}}{2 L}-6 \alpha_{6} \mu k_{3}-3 \alpha_{7} \mu k_{3}+\alpha_{8} \mu \frac{E A_{2}}{2 L}\right)}{\left(\alpha_{2}-\alpha_{5} \mu\right) \rho A_{2}}\left(\frac{I_{1}}{A_{1}}\right) \\
& g_{3}=\frac{c_{1}}{\rho A_{2}}
\end{aligned}
$$

$$
\begin{aligned}
& (1-p)\left\{\omega_{0}^{2}\left(\frac{d^{2} a_{2}}{d \tau^{2}}+a_{2}\right)\right\} \\
& +p\left\{\omega_{0}^{2} \frac{d^{2} a_{2}}{d \tau^{2}}+g_{1} a_{2}+g_{2} a_{2}^{3}-g_{3} a_{1}\right\}=0 .
\end{aligned}
$$

The solutions of $a_{1}=a_{1}(\tau, p), a_{2}=a_{2}(\tau, p)$ and $\omega=\omega(p)$ of the homotopy change from their initial approximations $a_{10}=a_{1}(\tau), a_{20}=a_{2}(\tau)$ and $\omega_{0}$ to the required solutions $a_{1}(\tau), a_{2}(\tau)$ and $\omega_{0}$ of Eqs. (49) and (50) as the embedding parameter $p$ travels from 0 to 1 .

Assuming the solution of Eqs. (45) and (46) to be in the following form

$a_{1}(\tau)=a_{10}(\tau)+p a_{11}(\tau)+p^{2} a_{12}(\tau)+p^{3} a_{13}(\tau)+\cdots$,

$a_{2}(\tau)=a_{20}(\tau)+p a_{21}(\tau)+p^{2} a_{22}(\tau)+p^{3} a_{23}(\tau)+\cdots$,

$\omega=\omega_{0}+p \omega_{1}+p^{2} \omega_{2}+p^{3} \omega_{3}+\cdots$

Substituting Eqs. $(45 \mathrm{a}-\mathrm{c})$ into the homotopy in Eqs. (44a) and (44b), collecting and rearranging the coefficients of the terms with identical powers of $p$, we have a series of linear differential equations

$p^{0}:\left\{\begin{array}{l}\frac{d^{2} a_{10}}{d \tau^{2}}+a_{10}=0, a_{10}(0)=X_{1}, \frac{d a_{10}(0)}{d \tau}=0, \\ \frac{d^{2} a_{20}}{d \tau^{2}}+a_{20}=0, a_{20}(0)=X_{1}, \frac{d a_{20}(0)}{d \tau}=0\end{array}\right.$ 
The solution of the initial zeroth approximation in Eq. (50a) is simply given by

$a_{10}(0)=X_{1} \cos \tau$,
$a_{20}(0)=X_{2} \cos \tau$,

Substituting Eqs. (51) and (52) into the first approximation in Eq. (50b), eliminating the coefficient of $\cos \tau$ in the above system to avoid the secular terms, we have the following nonlinear system of equations:

$-X_{1} \omega_{0}^{2}+f_{1} X_{1}+\frac{3}{4} f_{2} X_{1}^{3}-f_{3} X_{2}=0$

$-X_{2} \omega_{0}^{2}+g_{1} X_{2}+\frac{3}{4} g_{2} X_{2}^{3}-g_{3} X_{1}=0$

From Eq. (53),

$X_{2}=\frac{-X_{1} \omega_{0}^{2}+f_{1} X_{1}+\frac{3}{4} f_{2} X_{1}^{3}}{f_{3}}$.

After the substitution of Eq. (55) into Eq. (53), we have

$$
\begin{aligned}
& -\omega_{0}^{2}\left(\frac{-X_{1} \omega_{0}^{2}+f_{1} X_{1}+\frac{3}{4} f_{2} X_{1}^{3}}{f_{3}}\right)+g_{1}\left(\frac{-X_{1} \omega_{0}^{2}+f_{1} X_{1}+\frac{3}{4} f_{2} X_{1}^{3}}{f_{3}}\right) \\
& +\frac{3}{4} g_{2}\left(\frac{-X_{1} \omega_{0}^{2}+f_{1} X_{1}+\frac{3}{4} f_{2} X_{1}^{3}}{f_{3}}\right)^{3}-g_{3} X_{1}=0
\end{aligned}
$$

After expansion of Eq. (56) and collecting like terms, we arrived at

$$
\begin{gathered}
\frac{3}{4} \frac{g_{2}}{f_{3}^{3}} X_{1}^{3} \omega_{0}^{6}+\left(\frac{X_{1}}{f_{3}}-\frac{3}{4} \frac{g_{2}}{f_{3}^{3}} X_{1}^{2}\left(f_{1} X_{1}+\frac{3}{2} f_{2} X_{1}^{3}\right)\right. \\
\left.-\frac{9}{8} \frac{g_{2}}{f_{3}^{3}} f_{1} f_{2} X_{1}^{8}\right) \omega_{0}^{4}+\left(\frac{f_{1} X_{1}+\frac{3}{4} f_{2} X_{1}^{3}+g_{1} X_{1}}{f_{3}}\right. \\
+\frac{3}{4} \frac{g_{2}}{f_{3}^{3}} f_{1}^{2} X_{1}^{3}+\frac{9}{16} f_{2}^{2} X_{1}^{7}+2 X_{1}\left(f_{1}^{2} X_{1}^{2}+\frac{9}{16} f_{2}^{2} X_{1}^{6}\right) \\
\left.+\frac{9}{2} f_{1} f_{2} X_{1}^{5}\right) \omega_{0}^{2}+g_{3} X_{1}-g_{1}\left(\frac{f_{1} X_{1}+\frac{3}{4} f_{2} X_{1}^{3}}{f_{3}}\right) \\
\quad-\frac{3}{4} \frac{g_{2}}{f_{3}^{3}}\left\{f_{1}^{3} X_{1}^{3}+\frac{27}{64} f_{2}^{3} X_{1}^{6}+\frac{3}{4} f_{1}^{2} f_{2} X_{1}^{5}\right. \\
\left.+\frac{9}{16} f_{1} f_{2}^{2} X_{1}^{7}+2\left[\frac{3}{4} f_{1}^{2} f_{2} X_{1}^{5}+\frac{9}{16} f_{1} f_{2}^{2} X_{1}^{7}\right]\right\}=0
\end{gathered}
$$

Equation (57) can be written as

$$
\lambda_{1} \omega_{0}^{6}+\lambda_{2} \omega_{0}^{4}+\lambda_{3} \omega_{0}^{2}+\lambda_{4}=0
$$

where

$$
\begin{aligned}
\lambda_{1}= & \frac{3}{4} \frac{g_{2}}{f_{3}^{3}} X_{1}^{3} \\
\lambda_{2}= & \left(\frac{X_{1}}{f_{3}}-\frac{3}{4} \frac{g_{2}}{f_{3}^{3}} X_{1}^{2}\left(f_{1} X_{1}+\frac{3}{2} f_{2} X_{1}^{3}\right)-\frac{9}{8} \frac{g_{2}}{f_{3}^{3}} f_{1} f_{2} X_{1}^{8}\right) \\
\lambda_{3}= & \left(\frac{f_{1} X_{1}+\frac{3}{4} f_{2} X_{1}^{3}+g_{1} X_{1}}{f_{3}}+\frac{3}{4} \frac{g_{2}}{f_{3}^{3}} f_{1}^{2} X_{1}^{3}+\frac{9}{16} f_{2}^{2} X_{1}^{7}\right. \\
& \left.+2 X_{1}\left(f_{1}^{2} X_{1}^{2}+\frac{9}{16} f_{2}^{2} X_{1}^{6}\right)+\frac{9}{2} f_{1} f_{2} X_{1}^{5}\right) \\
\lambda_{4}= & g_{3} X_{1}-g_{1}\left(\frac{f_{1} X_{1}+\frac{3}{4} f_{2} X_{1}^{3}}{f_{3}}\right)-\frac{3}{4} \frac{g_{2}}{f_{3}^{3}}\left\{f_{1}^{3} X_{1}^{3}\right. \\
& +\frac{27}{64} f_{2}^{3} X_{1}^{6}+\frac{3}{4} f_{1}^{2} f_{2} X_{1}^{5}+\frac{9}{16} f_{1} f_{2}^{2} X_{1}^{7} \\
& \left.+2\left[\frac{3}{4} f_{1}^{2} f_{2} X_{1}^{5}+\frac{9}{16} f_{1} f_{2}^{2} X_{1}^{7}\right]\right\}
\end{aligned}
$$

The roots of the sexic equations are

$$
\begin{aligned}
& \left(\omega_{0}\right)_{1}=\sqrt{\begin{array}{l}
\sqrt[3]{\left(\frac{-\lambda_{2}^{3}}{27 \lambda_{1}^{3}}+\frac{\lambda_{2} \lambda_{3}}{6 \lambda_{1}^{2}}-\frac{\lambda_{4}}{2 \lambda_{1}}\right)+\sqrt{\left(\frac{\lambda_{3}}{3 \lambda_{1}}-\frac{\lambda_{2}^{2}}{9 \lambda_{1}^{2}}\right)^{3}+\left(\frac{-\lambda_{2}^{3}}{27 \lambda_{1}^{3}}+\frac{\lambda_{2} \lambda_{3}}{6 \lambda_{1}^{2}}-\frac{\lambda_{4}}{2 \lambda_{1}}\right)^{2}}} \\
+\sqrt[3]{\left(\frac{-\lambda_{2}^{3}}{27 \lambda_{1}^{3}}+\frac{\lambda_{2} \lambda_{3}}{6 \lambda_{1}^{2}}-\frac{\lambda_{4}}{2 \lambda_{1}}\right)-\sqrt{\left(\frac{\lambda_{3}}{3 \lambda_{1}}-\frac{\lambda_{2}^{2}}{9 \lambda_{1}^{2}}\right)^{3}+\left(\frac{-\lambda_{2}^{3}}{27 \lambda_{1}^{3}}+\frac{\lambda_{2} \lambda_{3}}{6 \lambda_{1}^{2}}-\frac{\lambda_{4}}{2 \lambda_{1}}\right)^{2}}}-\frac{\lambda_{2}}{3 \lambda_{1}}
\end{array}}
\end{aligned}
$$

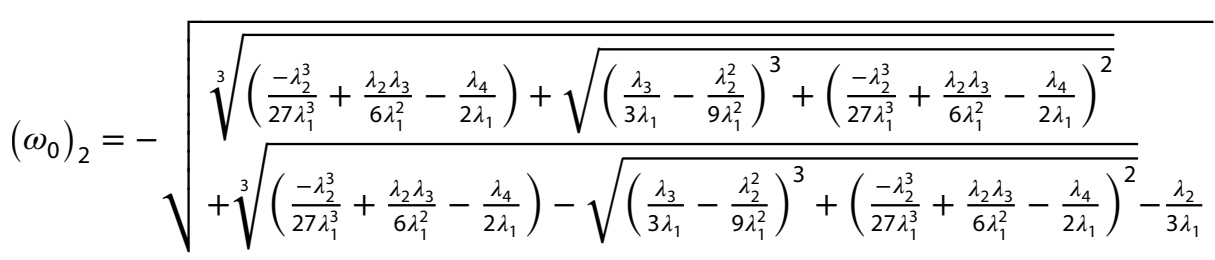




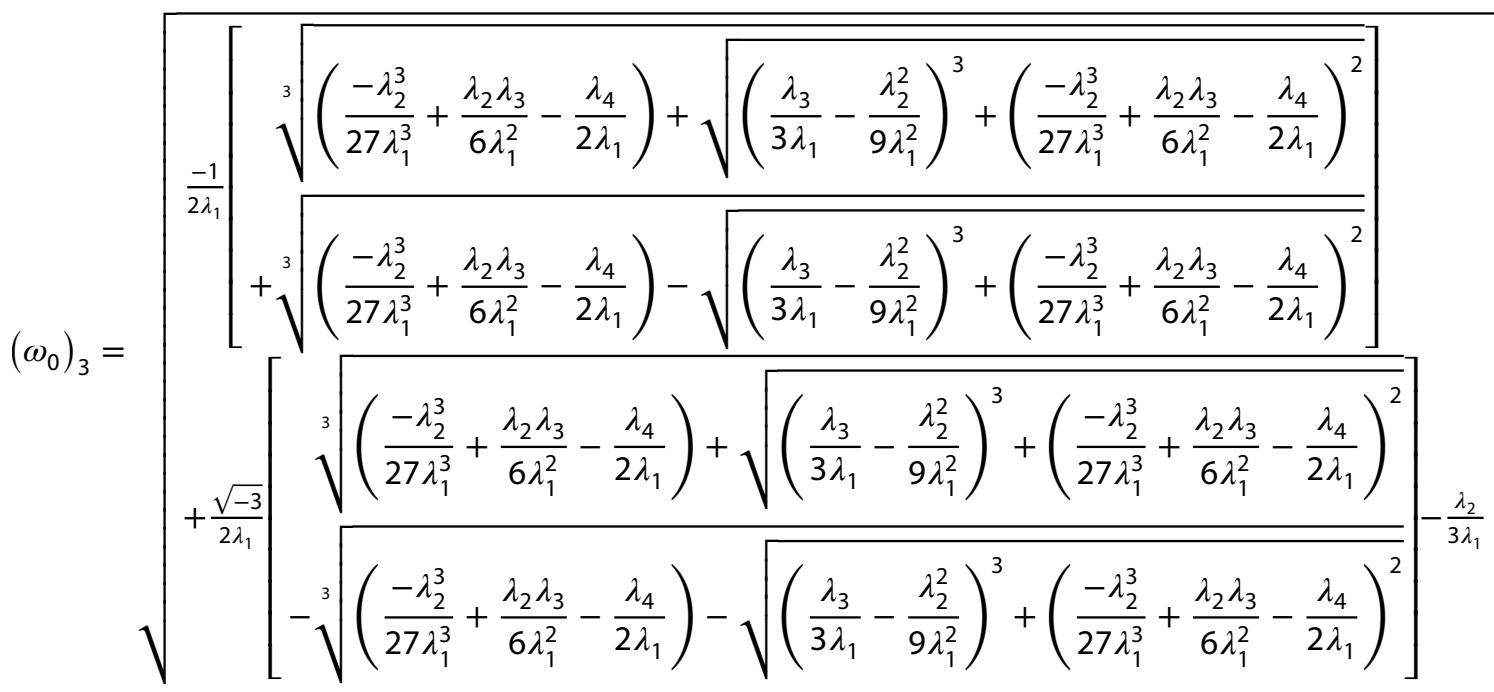

$(60 c)$

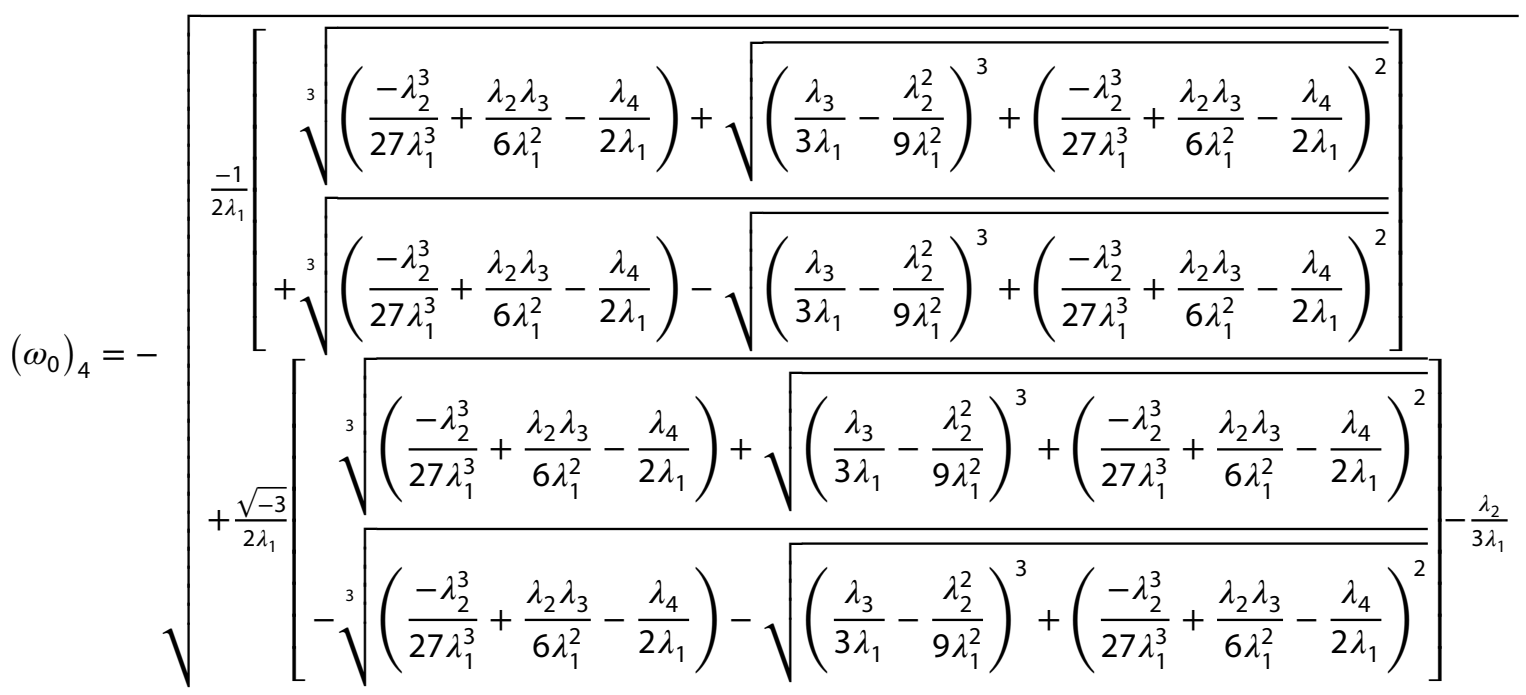

$(60 d)$ 


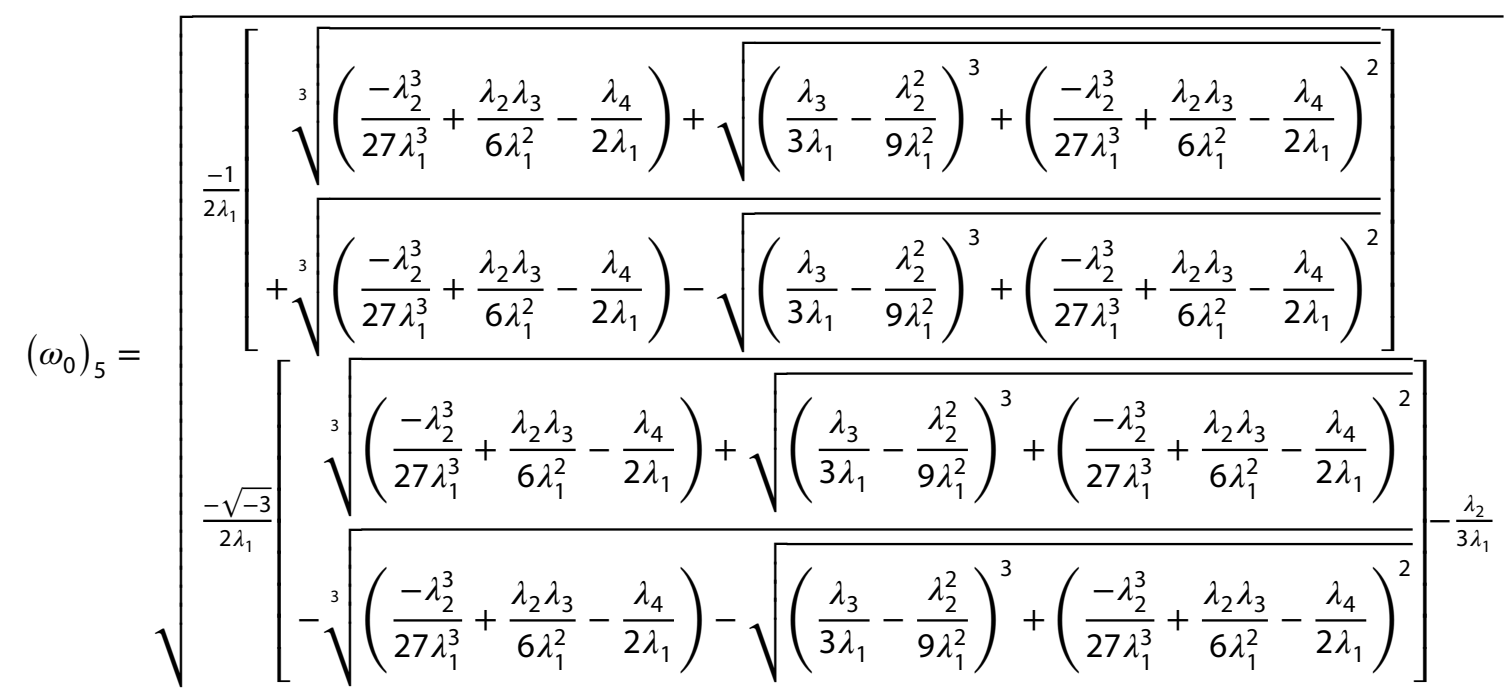

$(60 e)$

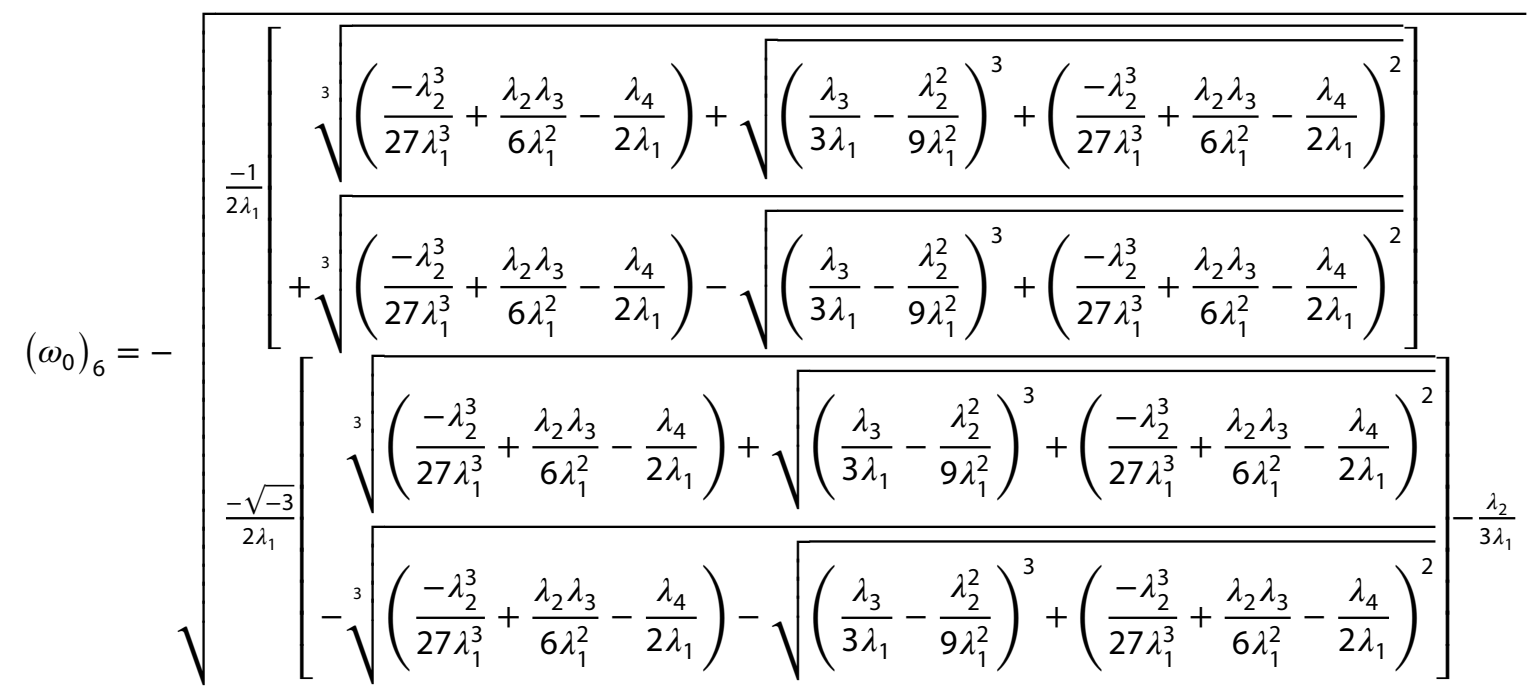

The nonlinear natural frequency $\left(\omega_{0}\right)$ for embedded DWCNTs is obtained from the above solution. The smallest real value of $\omega_{0}$ is the nonlinear natural frequency for DWCNTs. From Eq. (60),

$a_{10}(\tau)=X_{1} \cos \omega t$

$a_{20}(\tau)=X_{2} \cos \omega t$

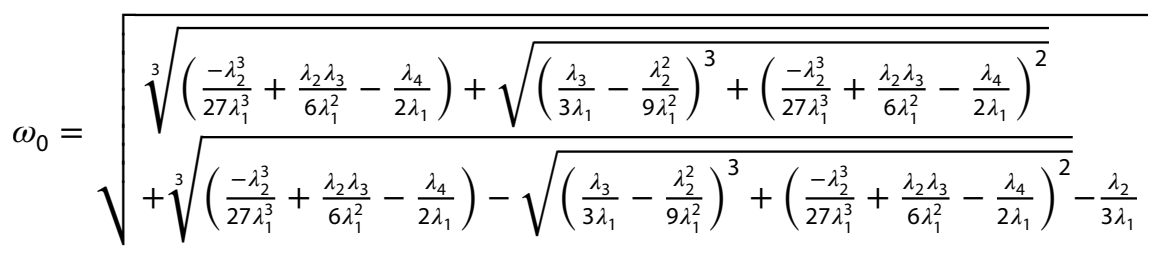

To calculate the linear natural frequencies for DWNT, substitute 
into Eq. (50b) and neglecting the nonlinear terms give

$-X_{1} \omega^{2}+f_{1} X_{1}-f_{3} X_{2}=0$

$-X_{2} \omega^{2}+g_{1} X_{2}-g_{3} X_{1}=0$

which can be written in matrix form as

$\left[\begin{array}{cc}-\omega^{2}+f_{1} & -f_{1} \\ -g_{3} & -\omega^{2}+g_{1}\end{array}\right]\left[\begin{array}{l}X_{1} \\ X_{2}\end{array}\right]=\left[\begin{array}{l}0 \\ 0\end{array}\right]$

Since $\left[\begin{array}{l}X_{1} \\ X_{2}\end{array}\right]$ cannot be equal to zero, for nontrivial case to occur, then

$\left[\begin{array}{cc}-\omega^{2}+f_{1} & -f_{1} \\ -g_{3} & -\omega^{2}+g_{1}\end{array}\right]=\left[\begin{array}{l}0 \\ 0\end{array}\right]$

By equating the determinant of the matrix in Eq. (66) to zero, the frequency characteristic equation is obtained as

$\omega^{4}-\left(f_{1}+g_{1}\right) \omega^{2}+f_{1} g_{1}-f_{3} g_{3}=0$

where the roots of the quartic equation are

$(\omega)_{1}=\sqrt{\frac{\left(f_{1}+g_{1}\right)+\sqrt{\left(f_{1}+g_{1}\right)^{2}-4\left(f_{1} g_{1}-f_{3} g_{3}\right)}}{2}}$ $(\omega)_{2}=-\sqrt{\frac{\left(f_{1}+g_{1}\right)+\sqrt{\left(f_{1}+g_{1}\right)^{2}-4\left(f_{1} g_{1}-f_{3} g_{3}\right)}}{2}}$

(68b)

$(\omega)_{3}=\sqrt{\frac{\left(f_{1}+g_{1}\right)-\sqrt{\left(f_{1}+g_{1}\right)^{2}-4\left(f_{1} g_{1}-f_{3} g_{3}\right)}}{2}}$

$(\omega)_{4}=-\sqrt{\frac{\left(f_{1}+g_{1}\right)-\sqrt{\left(f_{1}+g_{1}\right)^{2}-4\left(f_{1} g_{1}-f_{3} g_{3}\right)}}{2}}$

(68d)

The linear natural frequency of DWNTs is the lowest root of the Eq. (67). From Eq. (68), it is

$\omega=\sqrt{\frac{\left(f_{1}+g_{1}\right)-\sqrt{\left(f_{1}+g_{1}\right)^{2}-4\left(f_{1} g_{1}-f_{3} g_{3}\right)}}{2}}$

We should recall that frequency ratio is given by $\psi=\frac{\omega_{o}}{\omega}$. Therefore

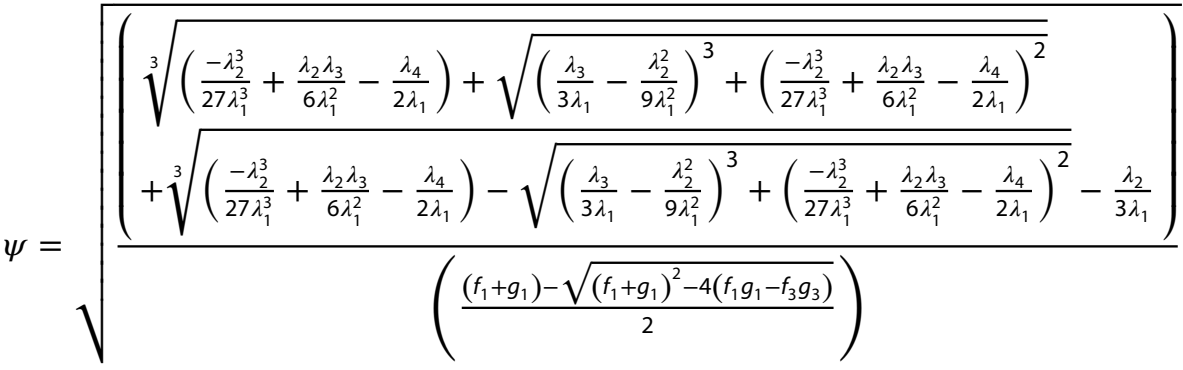

$\left\{\begin{array}{l}\omega_{0}^{2}\left\{\frac{d^{2} a_{11}}{d \tau^{2}}+a_{11}\right\}-\omega_{0}^{2} X_{1} \cos \tau+f_{1} X_{1} \cos \tau+f_{2}\left(X_{1} \cos \tau\right)^{3}-f_{3} X_{2} \cos \tau=0, \quad a_{11}(0)=0 \frac{d a_{11}(0)}{d \tau}=0, \\ \omega_{0}^{2}\left\{\frac{d^{2} a_{21}}{d \tau^{2}}+a_{21}\right\}-\omega_{0}^{2} X_{2} \cos \tau+g_{1} X_{2} \cos \tau+g_{2}\left(X_{2} \cos \tau\right)^{3}-g_{3} X_{1} \cos \tau=0, a_{21}(0)=0, \frac{d a_{21}(0)}{d \tau}=0\end{array}\right.$

After the application of trigonometry identities to the fourth-term in the LHS

$\left\{\begin{array}{lc}\omega_{0}^{2}\left\{\frac{d^{2} a_{11}}{d \tau^{2}}+a_{11}\right\}-\omega_{0}^{2} X_{1} \cos \tau+f_{1} X_{1} \cos \tau+\frac{3}{4} f_{2} X_{1}^{3} \cos \tau+\frac{1}{4} f_{2} X_{1}^{3} \cos 3 \tau-f_{3} X_{2} \cos \tau=0, \quad a_{11}(0)=0 \quad \frac{d a_{11}(0)}{d \tau}=0, \\ \omega_{0}^{2}\left\{\frac{d^{2} a_{21}}{d \tau^{2}}+a_{21}\right\}-\omega_{0}^{2} X_{2} \cos \tau+g_{1} X_{2} \cos \tau+\frac{3}{4} g_{2} X_{2}^{3} \cos \tau+\frac{1}{4} g_{2} X_{2}^{3} \cos 3 \tau-g_{3} X_{1} \cos \tau=0, \quad a_{21}(0)=0, \quad \frac{d a_{21}(0)}{d \tau}=0\end{array}\right.$ 
The solutions of Eqs. (72) are

$$
a_{11}(\tau)=\frac{f_{2} X^{3}(\cos 3 \tau-\cos \tau)}{32\left(\begin{array}{l}
\sqrt[3]{\left(\frac{-\lambda_{2}^{3}}{27 \lambda_{1}^{3}}+\frac{\lambda_{2} \lambda_{3}}{6 \lambda_{1}^{2}}-\frac{\lambda_{4}}{2 \lambda_{1}}\right)+\sqrt{\left(\frac{\lambda_{3}}{3 \lambda_{1}}-\frac{\lambda_{2}^{2}}{9 \lambda_{1}^{2}}\right)^{3}+\left(\frac{-\lambda_{2}^{3}}{27 \lambda_{1}^{3}}+\frac{\lambda_{2} \lambda_{3}}{6 \lambda_{1}^{2}}-\frac{\lambda_{4}}{2 \lambda_{1}}\right)^{2}}} \\
+\sqrt[3]{\left(\frac{-\lambda_{2}^{3}}{27 \lambda_{1}^{3}}+\frac{\lambda_{2} \lambda_{3}}{6 \lambda_{1}^{2}}-\frac{\lambda_{4}}{2 \lambda_{1}}\right)-\sqrt{\left(\frac{\lambda_{3}}{3 \lambda_{1}}-\frac{\lambda_{2}^{2}}{9 \lambda_{1}^{2}}\right)^{3}+\left(\frac{-\lambda_{2}^{3}}{27 \lambda_{1}^{3}}+\frac{\lambda_{2} \lambda_{3}}{6 \lambda_{1}^{2}}-\frac{\lambda_{4}}{2 \lambda_{1}}\right)^{2}}}-\frac{\lambda_{2}}{3 \lambda_{1}}
\end{array}\right)}
$$

$$
a_{21}(\tau)=\frac{g_{2} X^{3}(\cos 3 \tau-\cos \tau)}{32\left(\begin{array}{l}
\sqrt[3]{\left(\frac{-\lambda_{2}^{3}}{27 \lambda_{1}^{3}}+\frac{\lambda_{2} \lambda_{3}}{6 \lambda_{1}^{2}}-\frac{\lambda_{4}}{2 \lambda_{1}}\right)+\sqrt{\left(\frac{\lambda_{3}}{3 \lambda_{1}}-\frac{\lambda_{2}^{2}}{9 \lambda_{1}^{2}}\right)^{3}+\left(\frac{-\lambda_{2}^{3}}{27 \lambda_{1}^{3}}+\frac{\lambda_{2} \lambda_{3}}{6 \lambda_{1}^{2}}-\frac{\lambda_{4}}{2 \lambda_{1}}\right)^{2}}} \\
+\sqrt[3]{\left(\frac{-\lambda_{2}^{3}}{27 \lambda_{1}^{3}}+\frac{\lambda_{2} \lambda_{3}}{6 \lambda_{1}^{2}}-\frac{\lambda_{4}}{2 \lambda_{1}}\right)-\sqrt{\left(\frac{\lambda_{3}}{3 \lambda_{1}}-\frac{\lambda_{2}^{2}}{9 \lambda_{1}^{2}}\right)^{3}+\left(\frac{-\lambda_{2}^{3}}{27 \lambda_{1}^{3}}+\frac{\lambda_{2} \lambda_{3}}{6 \lambda_{1}^{2}}-\frac{\lambda_{4}}{2 \lambda_{1}}\right)^{2}}-\frac{\lambda_{2}}{3 \lambda_{1}}}
\end{array}\right)}
$$

Therefore, the first approximate solution of Eqs. (45) and (46) can be written as follows:

$$
\begin{aligned}
& a_{1}(x, t)=X_{1} \cos \tau+\frac{f_{2} X^{3}(\cos 3 \tau-\cos \tau)}{32\left(\begin{array}{l}
\sqrt[3]{\left(\frac{-\lambda_{2}^{3}}{27 \lambda_{1}^{3}}+\frac{\lambda_{2} \lambda_{3}}{6 \lambda_{1}^{2}}-\frac{\lambda_{4}}{2 \lambda_{1}}\right)+\sqrt{\left(\frac{\lambda_{3}}{3 \lambda_{1}}-\frac{\lambda_{2}^{2}}{9 \lambda_{1}^{2}}\right)^{3}+\left(\frac{-\lambda_{2}^{3}}{27 \lambda_{1}^{3}}+\frac{\lambda_{2} \lambda_{3}}{6 \lambda_{1}^{2}}-\frac{\lambda_{4}}{2 \lambda_{1}}\right)^{2}}} \\
+\sqrt[3]{\left(\frac{-\lambda_{2}^{3}}{27 \lambda_{1}^{3}}+\frac{\lambda_{2} \lambda_{3}}{6 \lambda_{1}^{2}}-\frac{\lambda_{4}}{2 \lambda_{1}}\right)-\sqrt{\left(\frac{\lambda_{3}}{3 \lambda_{1}}-\frac{\lambda_{2}^{2}}{9 \lambda_{1}^{2}}\right)^{3}+\left(\frac{-\lambda_{2}^{3}}{27 \lambda_{1}^{3}}+\frac{\lambda_{2} \lambda_{3}}{6 \lambda_{1}^{2}}-\frac{\lambda_{4}}{2 \lambda_{1}}\right)^{2}}}-\frac{\lambda_{2}}{3 \lambda_{1}}
\end{array}\right)} \\
& a_{2}(x, t)=X_{2} \cos \tau+\frac{g_{2} X^{3}(\cos 3 \tau-\cos \tau)}{32\left(\begin{array}{l}
\sqrt[3]{\left(\frac{-\lambda_{2}^{3}}{27 \lambda_{1}^{3}}+\frac{\lambda_{2} \lambda_{3}}{6 \lambda_{1}^{2}}-\frac{\lambda_{4}}{2 \lambda_{1}}\right)+\sqrt{\left(\frac{\lambda_{3}}{3 \lambda_{1}}-\frac{\lambda_{2}^{2}}{9 \lambda_{1}^{2}}\right)^{3}+\left(\frac{-\lambda_{2}^{3}}{27 \lambda_{1}^{3}}+\frac{\lambda_{2} \lambda_{3}}{6 \lambda_{1}^{2}}-\frac{\lambda_{4}}{2 \lambda_{1}}\right)^{2}}} \\
+\sqrt[3]{\left(\frac{-\lambda_{2}^{3}}{27 \lambda_{1}^{3}}+\frac{\lambda_{2} \lambda_{3}}{6 \lambda_{1}^{2}}-\frac{\lambda_{4}}{2 \lambda_{1}}\right)-\sqrt{\left(\frac{\lambda_{3}}{3 \lambda_{1}}-\frac{\lambda_{2}^{2}}{9 \lambda_{1}^{2}}\right)^{3}+\left(\frac{-\lambda_{2}^{3}}{27 \lambda_{1}^{3}}+\frac{\lambda_{2} \lambda_{3}}{6 \lambda_{1}^{2}}-\frac{\lambda_{4}}{2 \lambda_{1}}\right)^{2}}}-\frac{\lambda_{2}}{3 \lambda_{1}}
\end{array}\right)}
\end{aligned}
$$

The displacements of the nanotubes to be expressed as for simple simply support as

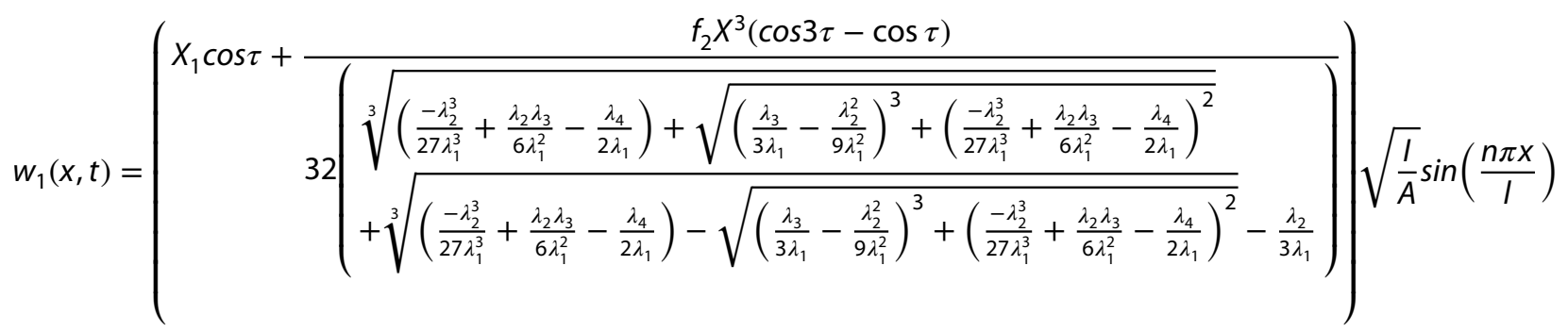




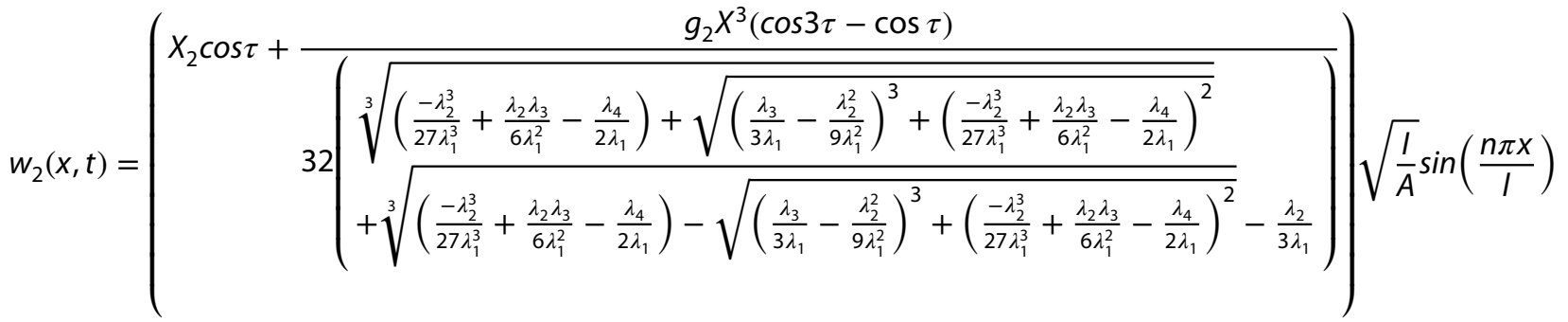

while for clamped-clamped are given as

$w_{1}(x, t)=\left(\begin{array}{l}x_{1} \cos \tau+\frac{f_{2} X^{3}(\cos 3 \tau-\cos \tau)}{32\left(+\sqrt[3]{\left(\frac{-\lambda_{2}^{3}}{27 \lambda_{1}^{3}}+\frac{\lambda_{2} \lambda_{3}}{6 \lambda_{1}^{2}}-\frac{\lambda_{4}}{2 \lambda_{1}}\right)+\sqrt{\left(\frac{\lambda_{3}}{3 \lambda_{1}}-\frac{\lambda_{2}^{2}}{9 \lambda_{1}^{2}}\right)^{3}+\left(\frac{-\lambda_{2}^{3}}{27 \lambda_{1}^{3}}+\frac{\lambda_{2} \lambda_{3}}{6 \lambda_{1}^{2}}-\frac{\lambda_{4}}{2 \lambda_{1}}\right)^{2}}}\right)} \\ \left.+\sqrt[3]{\left.\left(\frac{-\lambda_{2}^{3}}{27 \lambda_{1}^{3}}+\frac{\lambda_{2} \lambda_{3}}{6 \lambda_{1}^{2}}-\frac{\lambda_{4}}{2 \lambda_{1}}\right)-\sqrt{\left(\frac{\lambda_{3}}{3 \lambda_{1}}-\frac{\lambda_{2}^{2}}{9 \lambda_{1}^{2}}\right)^{3}+\left(\frac{-\lambda_{2}^{3}}{27 \lambda_{1}^{3}}+\frac{\lambda_{2} \lambda_{3}}{6 \lambda_{1}^{2}}-\frac{\lambda_{4}}{2 \lambda_{1}}\right)^{2}}-\frac{\lambda_{2}}{3 \lambda_{1}}\right)}\right)\end{array}\right.$

$\times \sqrt{\frac{I}{A}}\left\{\begin{array}{l}\left(\cosh \left(\frac{\beta x}{L}\right)-\cos \left(\frac{\beta x}{L}\right)\right) \\ -\left(\frac{\sinh \beta+\sin \beta}{\cosh \beta-\cos \beta}\right)\left(\sinh \left(\frac{\beta x}{L}\right)-\sin \left(\frac{\beta x}{L}\right)\right)\end{array}\right\}$

$w_{2}(x, t)=\left(\begin{array}{l}\left.x_{2} \cos \tau+\frac{g_{2} X^{3}(\cos 3 \tau-\cos \tau)}{32\left(\begin{array}{l}\sqrt[3]{\left(\frac{-\lambda_{2}^{3}}{27 \lambda_{1}^{3}}+\frac{\lambda_{2} \lambda_{3}}{6 \lambda_{1}^{2}}-\frac{\lambda_{4}}{2 \lambda_{1}}\right)+\sqrt{\left(\frac{\lambda_{3}}{3 \lambda_{1}}-\frac{\lambda_{2}^{2}}{9 \lambda_{1}^{2}}\right)^{3}+\left(\frac{-\lambda_{2}^{3}}{27 \lambda_{1}^{3}}+\frac{\lambda_{2} \lambda_{3}}{6 \lambda_{1}^{2}}-\frac{\lambda_{4}}{2 \lambda_{1}}\right)^{2}}} \\ +\sqrt[3]{\left(\frac{-\lambda_{2}^{3}}{27 \lambda_{1}^{3}}+\frac{\lambda_{2} \lambda_{3}}{6 \lambda_{1}^{2}}-\frac{\lambda_{4}}{2 \lambda_{1}}\right)-\sqrt{\left(\frac{\lambda_{3}}{3 \lambda_{1}}-\frac{\lambda_{2}^{2}}{9 \lambda_{1}^{2}}\right)^{3}+\left(\frac{-\lambda_{2}^{3}}{27 \lambda_{1}^{3}}+\frac{\lambda_{2} \lambda_{3}}{6 \lambda_{1}^{2}}-\frac{\lambda_{4}}{2 \lambda_{1}}\right)^{2}}-\frac{\lambda_{2}}{3 \lambda_{1}}}\end{array}\right)}\right)\end{array}\right)$

$\times \sqrt{\frac{I}{A}}\left\{\begin{array}{l}\left(\cosh \left(\frac{\beta x}{L}\right)-\cos \left(\frac{\beta x}{L}\right)\right) \\ -\left(\frac{\sinh \beta+\sin \beta}{\cosh \beta-\cos \beta}\right)\left(\sinh \left(\frac{\beta x}{L}\right)-\sin \left(\frac{\beta x}{L}\right)\right)\end{array}\right\}$ 
And for clamped-simply supports, we have

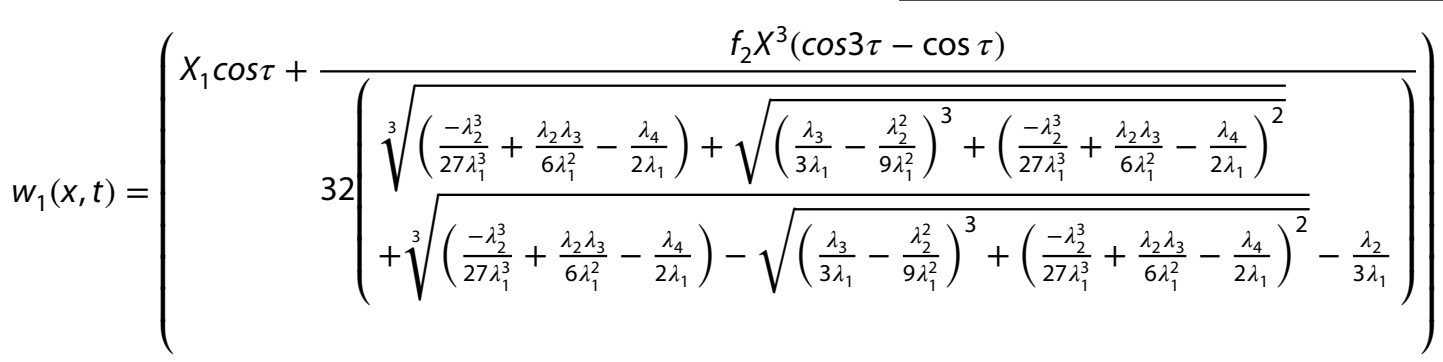

$$
\begin{aligned}
& \times \sqrt{\frac{I}{A}}\left\{\begin{array}{l}
\left(\cosh \left(\frac{\beta x}{L}\right)-\cos \left(\frac{\beta x}{L}\right)\right) \\
-\left(\frac{\cosh \beta-\cos \beta}{\sinh \beta-\sin \beta}\right)\left(\sinh \left(\frac{\beta x}{L}\right)-\sin \left(\frac{\beta x}{L}\right)\right)
\end{array}\right\}
\end{aligned}
$$

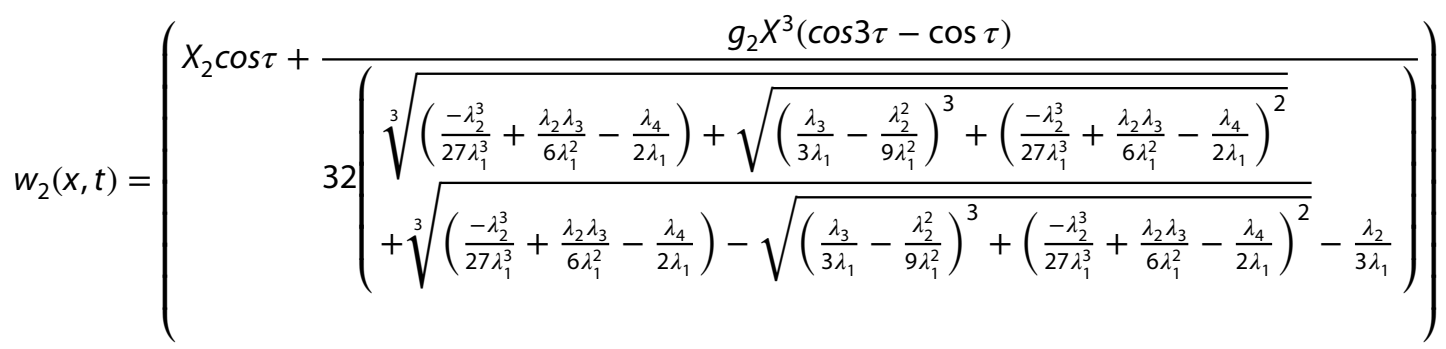

$$
\begin{aligned}
& \times \sqrt{\frac{I}{A}}\left\{\begin{array}{l}
\left(\cosh \left(\frac{\beta x}{L}\right)-\cos \left(\frac{\beta x}{L}\right)\right) \\
-\left(\frac{\cosh \beta-\cos \beta}{\sinh \beta-\sin \beta}\right)\left(\sinh \left(\frac{\beta x}{L}\right)-\sin \left(\frac{\beta x}{L}\right)\right)
\end{array}\right\}
\end{aligned}
$$

\section{Results and discussion}

Using the material and geometric parameters of the carbon nanotubes, $E=1.1 \mathrm{TPa}, \rho=1300 \mathrm{~kg} / \mathrm{m}^{3}, I=45 \mathrm{~nm}$, the outer diameter $d o=3 \mathrm{~nm}$, and the thickness of each layer, $h=0.68 \mathrm{~nm}$, the frequency ratio against non-dimensional maximum amplitude for the nonlinear vibrations of SWCNTs and DWCNTs in a thermal and magnetic environment are given in Figs. 3, 4, 5, 6, 7, 8, 9 and 10. The results of the simulation and the effects of various parameters on the frequency ratio of nonlinear vibrations of embedded single- and double-walled carbon nanotubes in a thermal and magnetic environment are presented and discussed.

\subsection{Effects of boundary conditions on the frequency ratio of the carbon nanotubes}

Figures 3 and 4 show the effects of boundary conditions on the frequency ratio for the nonlinear vibrations of SWCNTs and DWCNTs, respectively in thermal and magnetic environment $\left(k_{1}=10^{7} \mathrm{~N} / \mathrm{m}^{2}, k_{3}=10^{8} \mathrm{~N} / \mathrm{m}^{2}, T=40 \mathrm{~K}\right.$, $H_{x}=10^{7} \mathrm{~A} / \mathrm{m}, e_{0} a=1.5 \times 10^{-9}$ and $c_{1}=c_{2}=c_{3}=0.3 \times 10^{12} \mathrm{~N} /$ $\mathrm{m}^{2}$ ). As it is depicted in the figures, the frequency ratio for all boundary conditions decreases as the number of wall increases. This is due to the fact that carbon nanotubes generally have weak shear interactions between adjacent tubes and become more predominant as the number of walls increases. It could therefore be inferred that in an application where linear vibration is preferred for system stability, DWCNTs will perform better than SWCNTs of the same geometry and size. Also, the figures show that for both the SWCNTs and DWCNTs, the frequency ratio is highest for clamped simple supported and least for clamped-clamped supported. This establishes that the clamped-clamped supported system provided the best grip (support) for the nanotubes and this can be used to control nonlinear vibration of the system.

\subsection{Effects of spring stiffness $\left(k_{1}\right)$ on the frequency ratio of the carbon nanotubes}

The impacts of the spring stiffness $\left(k_{1}\right)$ on the dimensionless frequency ratio of the single- and double-walled carbon nanotubes in thermal and magnetic environment are shown in Fig. 5 and 6 . It is depicted that he frequency ratio decreases with increases in the value of spring constant $\left(k_{1}\right)$ for CNTs. This is because, the linear frequency increases as the value spring constant increases. At large value of 
Fig. 3 Frequency ratio versus non-dimensional amplitude for SWCNT under various boundary conditions
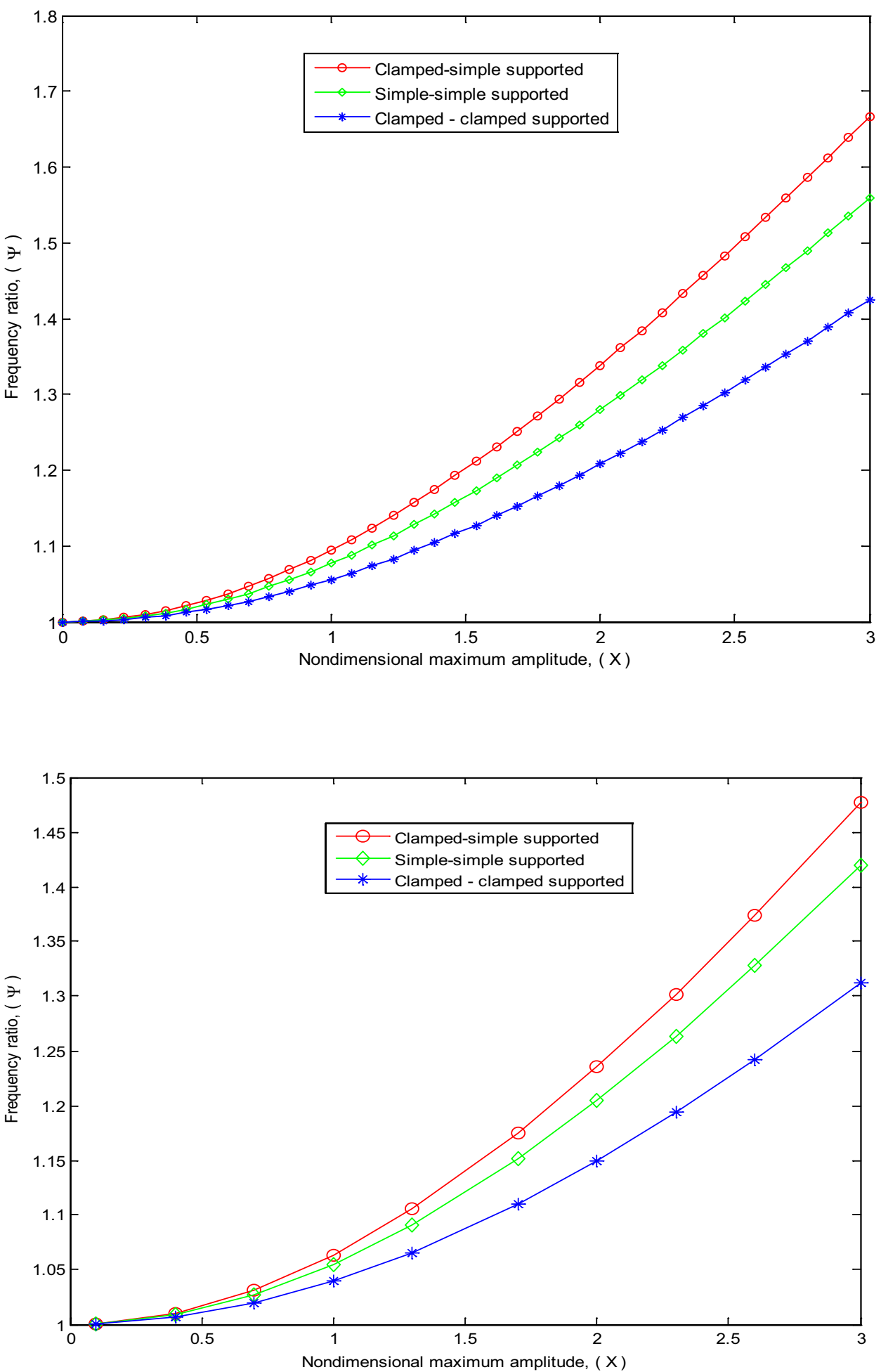

Fig. 4 Frequency ratio versus non-dimensional amplitude for DWCNT under various boundary conditions $k_{1}\left(k_{1}=10^{10} \mathrm{~N} / \mathrm{m}^{2}\right)$, the vibration of the system becomes stable and this can be used as good measure in controlling nonlinear vibration of the system.

\subsection{Effects of nonlinear spring stiffness $\left(k_{3}\right)$ on the frequency ratio of the carbon nanotubes}

Figure 7 shows the effect of nonlinear spring stiffness $\left(k_{3}\right)$ on the frequency ratio of outer walled of embedded DWCNTs in a thermal and magnetic environment. It could be 
Fig. 5 Effect of Winkler constant (k1) on amplitude-frequency ratio curve for SWCNT

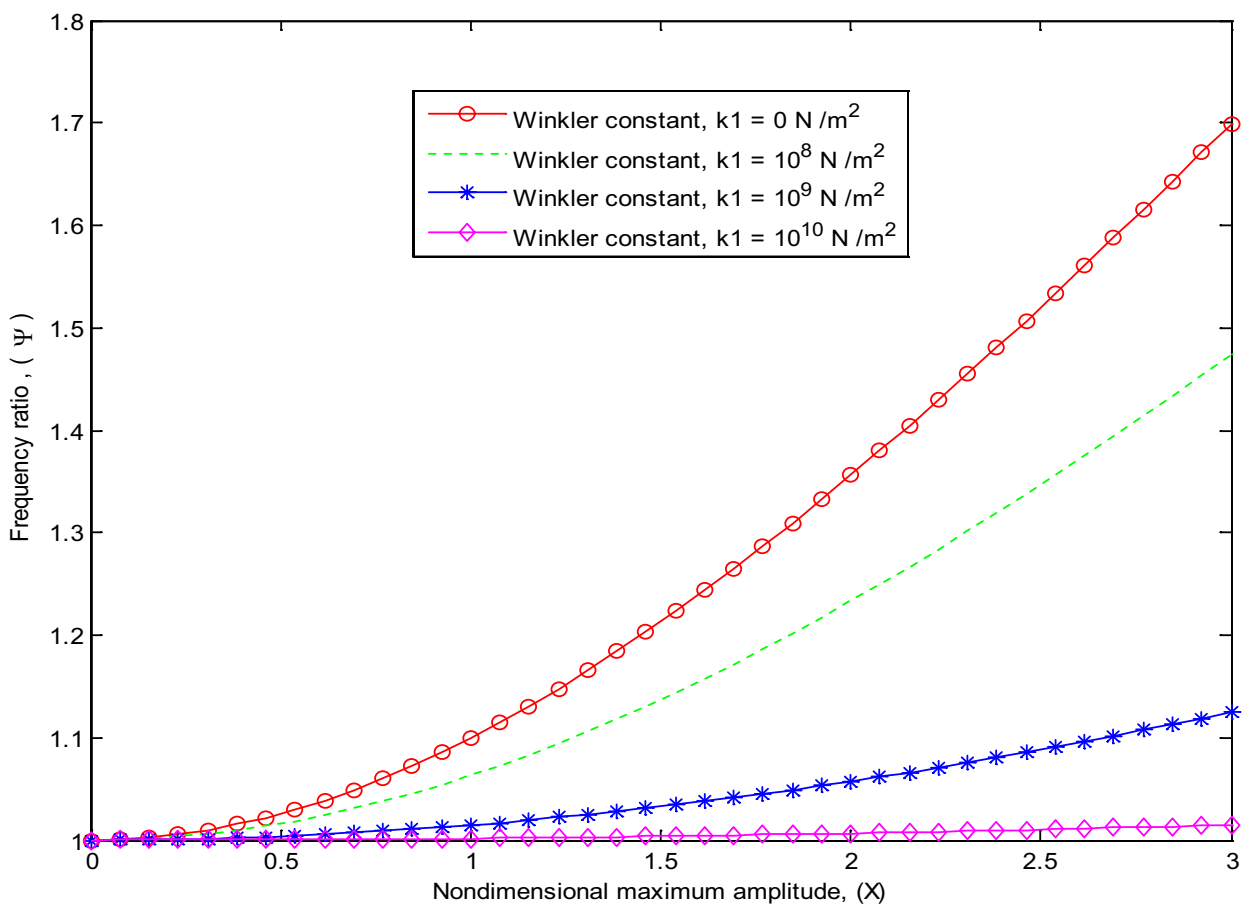

Fig. 6 Effect of Winkler constant $\left(\mathrm{k}_{1}\right)$ on amplitude-frequency ratio curve for DWCNTs

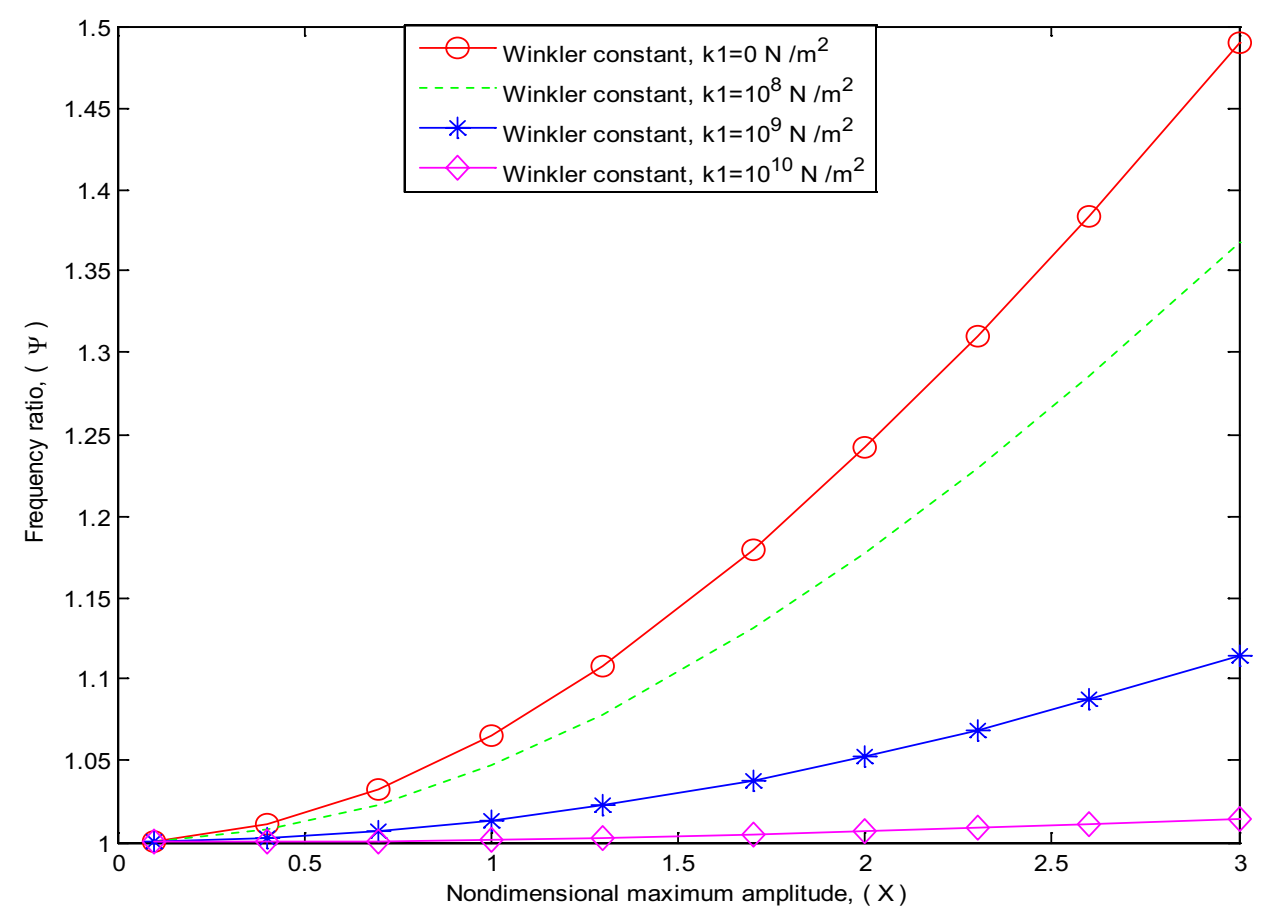

seen that the frequency ratio increases with increases in the value of the nonlinear spring constant. This is because the nonlinear frequency increases as the value of the nonlinear spring constant increases without producing any effect on the linear frequency. The value of nonlinear spring constant should be kept as low as possible since it makes the vibration of the system to be nonlinear and this can lead to the instability of the system. 
Fig. 7 Effect of nonlinear spring constant constant $\left(\mathrm{k}_{3}\right)$ on amplitude-frequency ratio curve for DWCNTs

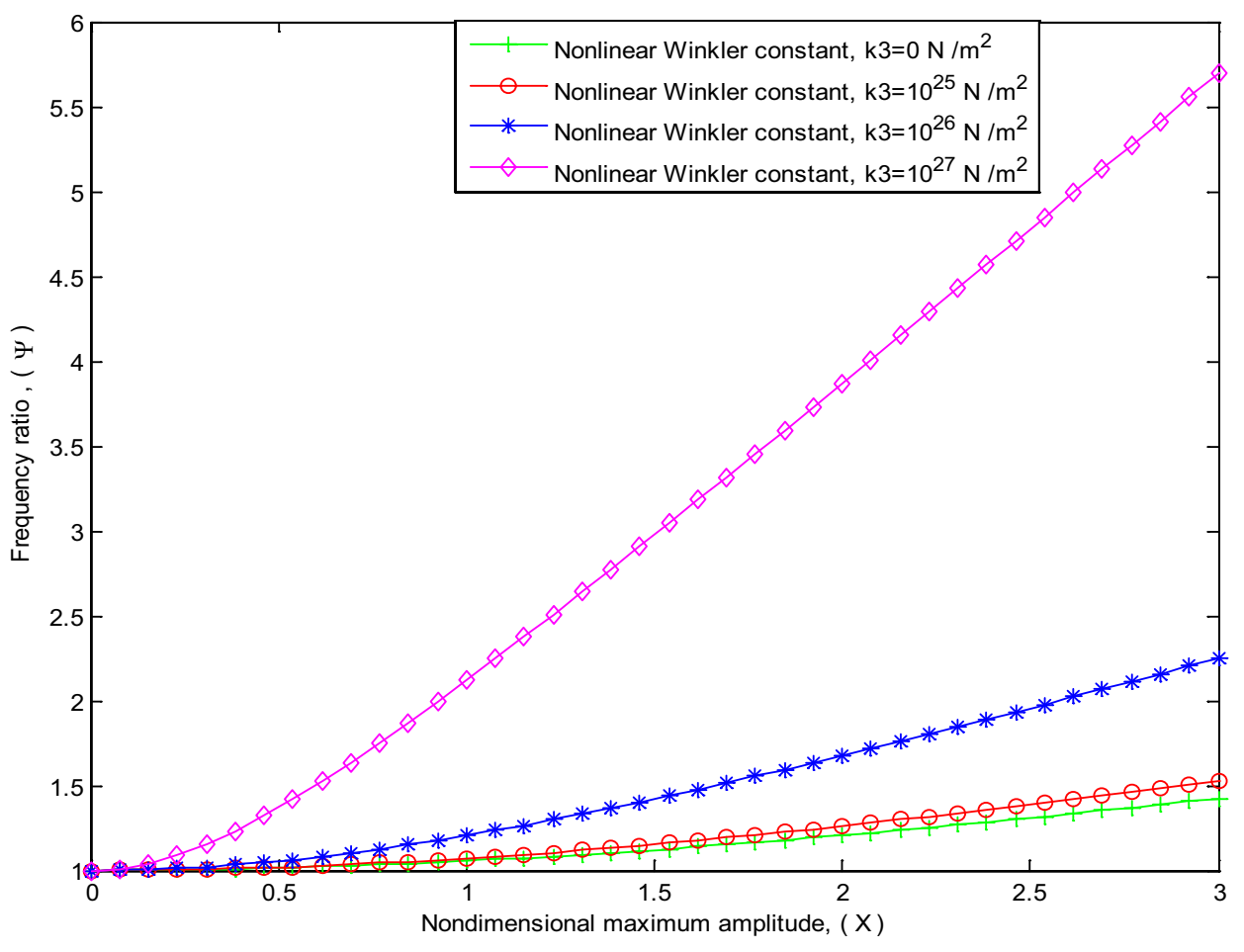

Fig. 8 Effect of Van der waals force on amplitude-frequency ratio curve for DWCNTs

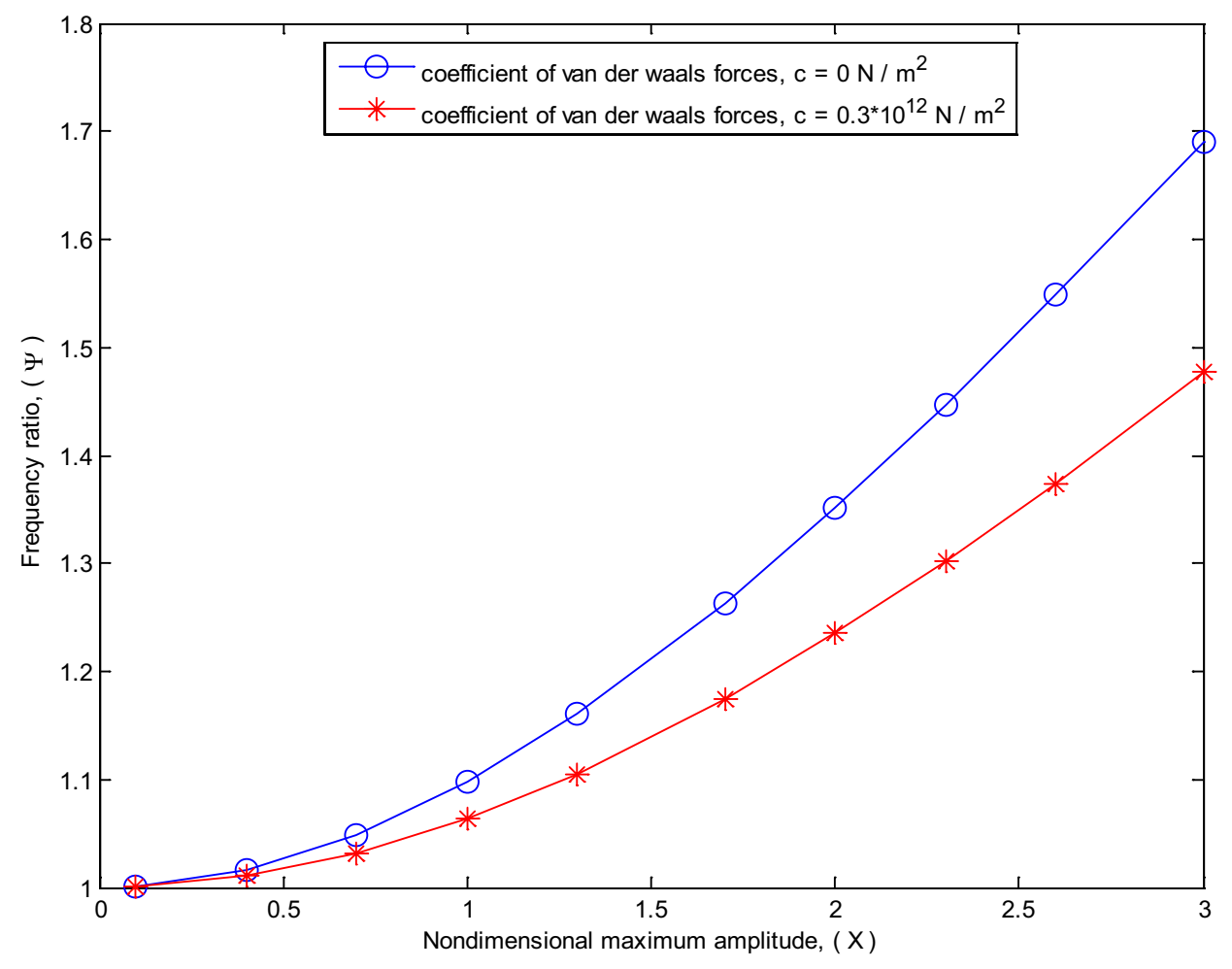

\subsection{Effects of Van der Waal force on the frequency ratio of the carbon nanotubes}

Figure 8 presents the effects of Van der Waal force on the frequency ratio of the SWCNTs and DWCNTs, respectively, in a thermal and magnetic environment. It can be seen that when the coefficient of the van der Waals forces is zero i.e. $c=0 \mathrm{~N} / \mathrm{m}^{2}$, it means a single-walled carbon nanotube with the same dimension and geometry with double-walled carbon nanotubes. The results reveals that the frequency 
Fig. 9 Effect of temperature on amplitude-frequency ratio curve on outerwall of DWCNTs
Fig. 10 Effect of magnetic force strength on amplitudefrequency ratio curve on outerwall of DWCNTs
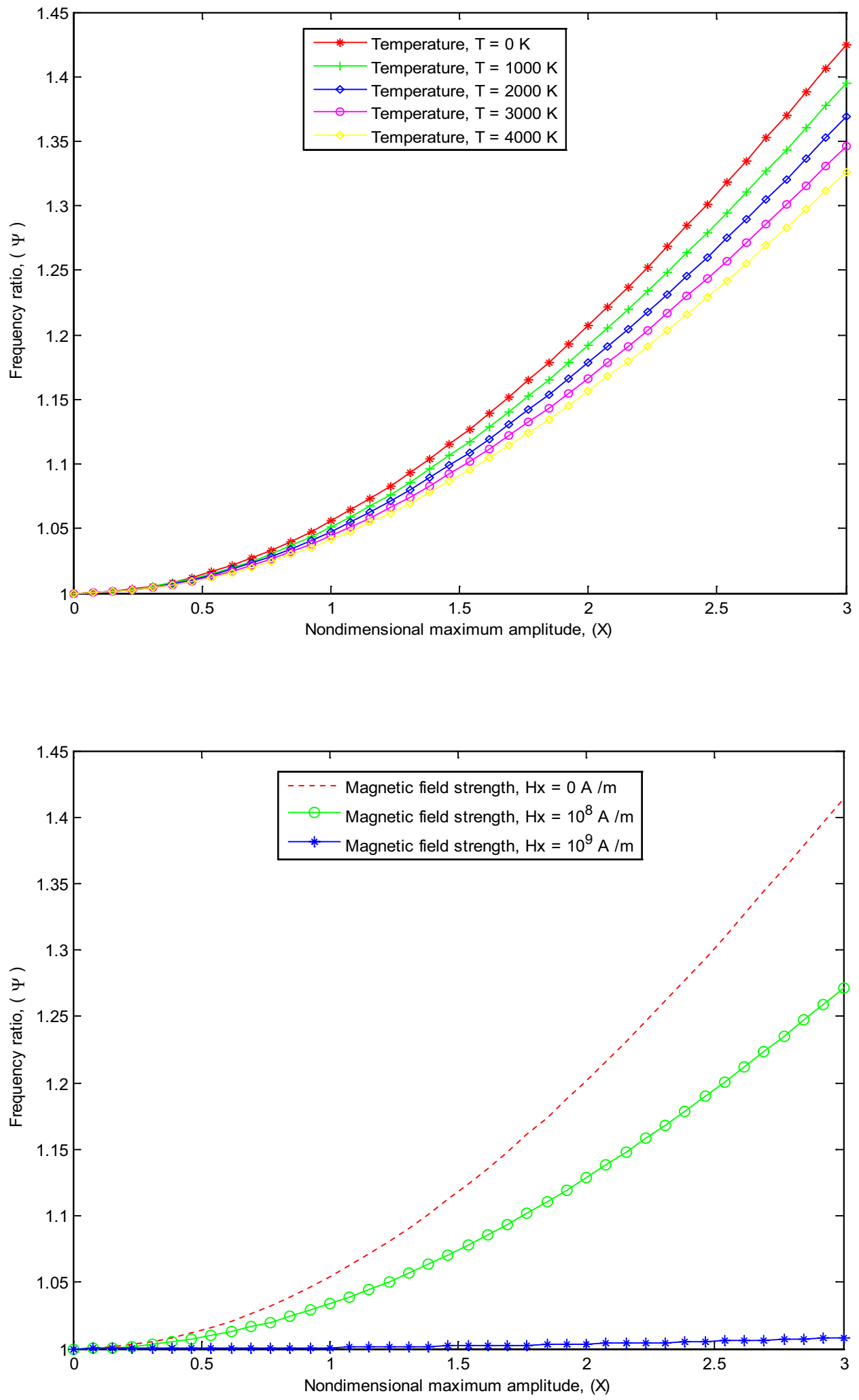
Fig. 11 The linear and nonlinear vibration deflection-time curve of outer wall of DWCNTs

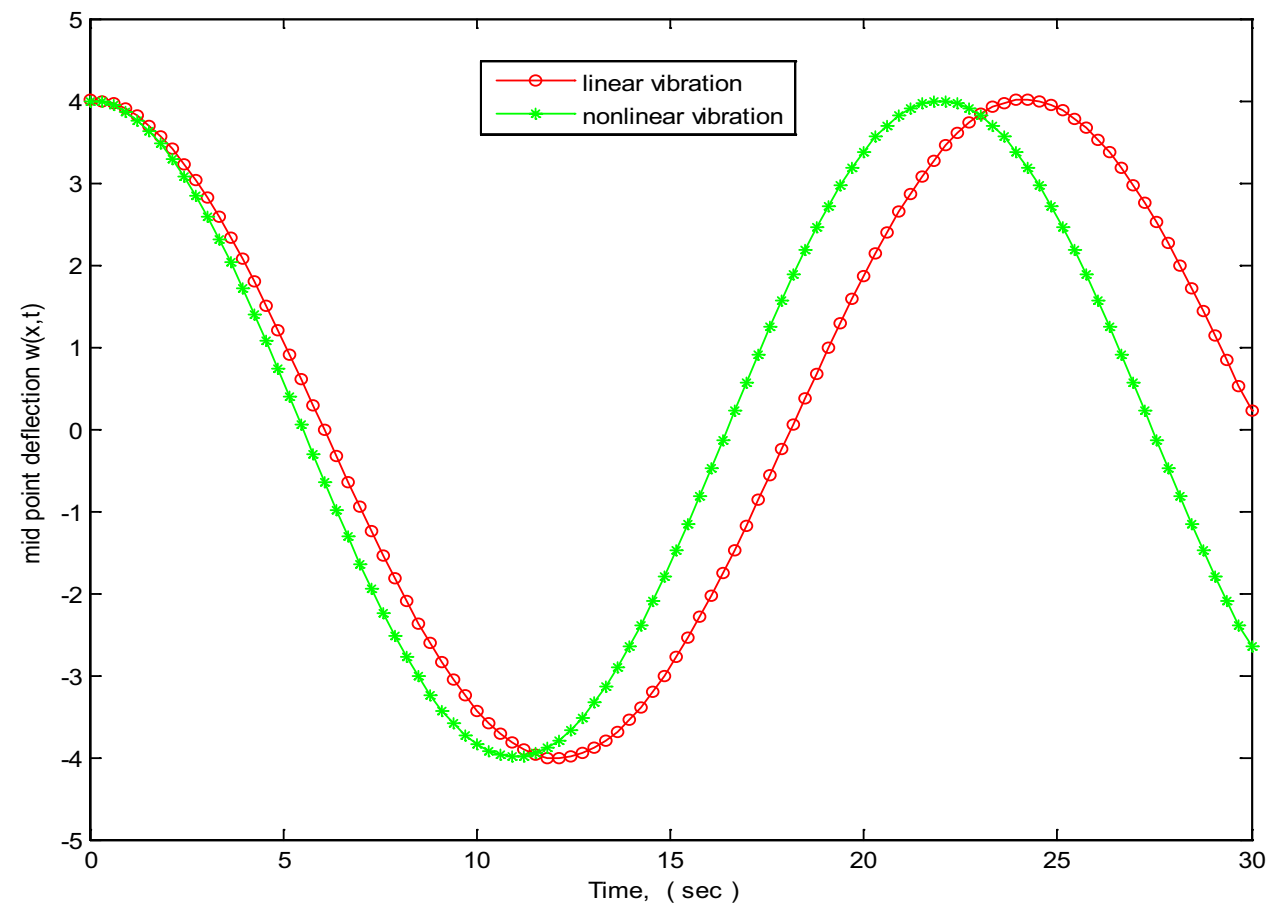

ratio decreases as the number of walls increases. Increasing the number of walls can be used as a good parameter to control the nonlinear vibration of the system.

\subsection{Effects of temperature on the frequency ratio of outer wall of DWCNTs}

Figure 9 illustrates the influence of temperature on the frequency ratio on the outer wall of DWCNTs in a thermal and magnetic environment. The result presents that as the temperature increases, the frequency ratio decreases. This shows that increase in temperature leads to increase in the value of linear natural frequency of the system.

\subsection{Effects of magnetic force strength on the frequency ratio of outer wall of DWCNTs}

Figure 10 presents the impact of magnetic force strength on the dimensionless frequency ratio. From the figure, it is established that as the magnetic field strength increases, the vibration of the system approaches linear vibration and become linear at higher value of magnetic force strength, $H=10^{9} \mathrm{~A} / \mathrm{m}$.

\subsection{The linear and nonlinear vibration deflection-time curve of outer wall of DWCNTs}

Figure 11 shows the comparison of the linear vibration with nonlinear vibration of the DWCNT. It could be seen in the figure that the discrepancy between the linear and nonlinear amplitudes increases and the vibration time progresses.

\section{Conclusion}

In this work, nonlocal elasticity theory has been used to analyze the nonlinear vibrations of single and doublewalled carbon nanotubes resting on two-parameter foundation in a thermal and magnetic environment. The effects of the various controlling parameters on the nonlinear to linear frequency ratio have been analyzed and discussed. The results established that the frequency ratio for all boundary conditions decreases as the number of walls increases from single to double. Also, it is established that the frequency ratio is highest for clamped-simple supported and least for clamped-clamped supported. Additionally, the results revealed that the frequency ratio decreases with increase in the value of spring constant $\left(k_{1}\right)$ temperature and magnetic field strength. This work will enhance the applications of carbon nanotubes in structural, electrical, mechanical and biological applications especially in a thermal and magnetic environment.

Acknowledgement This research was supported by University of Lagos, Nigeria under the research Grant CRC No. 2019/07. 


\section{Compliance with ethical standards}

Conflict of interest The authors declare that there is no conflict of interest regarding the publication of this research work.

\section{References}

1. lijima S (1991) Helical micro tubes of graphitic carbon. Nature 354:56-58

2. Terrones M, Banhart F, Grobert N, Charlier J, Terrones C, Ajayan $H$ (2002) Molecular junctions by joining single-walled carbon nanotubes. Phys Rev Lett 89:07550

3. Nagy P, Ehlich R, Biro LP, Gjyulai J (2000) Y-branching of single walled carbon nanotubes. Appl Phys A Mater 70:481-483

4. Chernozatonskii LA (1992) Carbon nanotubes connectors and planar jungle gyms. Appl Phys A 172:173-176

5. Liew KM, Wong CH, He XQ, Tan MJ, Meguid SA (2004) Nanomechanics of single and multiwalled carbon nanotubes. Phys Rev B 69:115429

6. Pantano A, Boyce MC, Parks DM (2004) Mechanics of axial compression of single and multi-wall carbon nanotubes. J Eng Mater Technol 126:279-284

7. Pantano A, Parks DM, Boyce MC (2004) Mechanics of deformation of single- and multi-wall carbon nanotubes. J Mech Phys Solids 52:789-821

8. Qian D, Wagner GJ, Liu WK, Yu MF, Ruoff RS (2002) Mechanics of carbon nanotubes. Appl Mech Rev 55:495-533

9. Salvetat JP, Bonard J-M, Thomson NH, Kulik AJ, Forro L, Benoit W, Zuppiroli $L$ (1999) Mechanical properties of carbon nanotubes. Appl Phys A 69:255-260

10. Sears A, Batra RC (2006) Buckling of carbon nanotubes under axial compression. Phys Rev B 73:085410

11. Yoon J, Ru CQ, Mioduchowski A (2002) Noncoaxial resonance of an isolated multiwall carbon nanotube. Phys Rev B 66:233402

12. Wang $X$, Cai $H$ (2006) Effects of initial stress on non-coaxial resonance of multi-wall carbon nanotubes. Acta Mater 54:2067-2074

13. Wang CM, Tan VBC, Zhang YY (2006) Timoshenko beam model for vibration analysis of multi-walled carbon nanotubes. J Sound Vib 294:1060-1072

14. Zhang Y, Liu G, Han X (2005) Transverse vibrations of doublewalled carbon nanotubes under compressive axial load. Phys Lett A 340:258-266

15. Elishakoff I, Pentaras D (2009) Fundamental natural frequencies of double-walled carbon nanotubes. J Sound Vib 322:652-664

16. Buks $E$, Yurke B (2006) Mass detection with nonlinear nanomechanical resonator. Phys Rev E 74:046619

17. Postma HWC, Kozinsky I, Husain A, Roukes ML (2005) Dynamic range of nanotube- and nanowire-based electromechanical systems. Appl Phys Lett 86:223105

18. Fu YM, Hong JW, Wang XQ (2006) Analysis of nonlinear vibration for embedded carbon nanotubes. J Sound Vib 296:746-756

19. Xu KY, Guo XN, Ru CQ (2006) Vibration of a double-walled carbon nanotube aroused by nonlinear intertube van der Waals forces. J Appl Phys 99:064303

20. Dequesnes M, Tang Z, Aluru NR (2004) Static and dynamic analysis of carbon nanotube-based switches. Trans ASME 126:230-237

21. Ouakad HM, Younis MI (2010) Nonlinear dynamics of electrically actuated carbon nanotube resonators. J Comput Nonlinear Dyn 5:011009
22. Zamanian M, Khadem SE, Mahmoodi SN (2009) Analysis of nonlinear vibrations of a microresonator under piezoelectric and electrostatic actuations. J Mech Eng Sci 223:329-344

23. Abdel-Rahman EM, Nayfeh AH (2003) Secondary resonances of electrically actuated resonant microsensors. J Micromech Microeng 13:491-501

24. Hawwa MA, Al-Qahtani HM (2010) Nonlinear oscillations of a double-walled carbon nanotube. Comput Mater Sci 48:140-143

25. Hajnayeb A, Khadem SE (2012) Nonlinear vibration and stability analysis of a double-walled carbon nanotube under electrostatic actuation. J Sound Vib 331:2443-2456

26. Belhadj A, Boukhalfa A, Belalia S (2016) Carbon nanotube structure vibration based on non local elasticity. J Mod Mater 3(1):913. https://doi.org/10.21467/jmm.3.1.9-13

27. Lei XW, Natsuki T, Shi JX, Ni QQ (2012) Surface effects on the vibrational frequency of double-walled carbon nanotubes using the nonlocal Timoshenko beam model. Compos B 43:64-69

28. Sharabiani PA, Yazdi MRH (2013) Nonlinear free vibrations of functionally graded nanobeams with surface effects. Compos B 45:581-586

29. Wang $L$ (2010) Vibration analysis of fluid-conveying nanotubes with consideration of surface effects. Physica E 43:437-439

30. Biro LP, Horvat ZE, Mark GI, Osvath Z, Koos AA, Santucci S, Kenny JM (2004) Carbon nanotube Y junctions: growth and properties. Diam Relat Mater 13:241-249

31. Lin RM (2012) Nanoscale vibration characterization of multi-layered graphene sheets embedded in an elastic medium. Comput Mater Sci 53:44-52

32. Pradhan SC, Phadikar JK (2009) Small scale effect on vibration of embedded multilayered graphene sheets based on nonlocal continuum models. Phys Lett A 373:1062-1069

33. Ghorbanpour AA, Zarei MS, Amir S, Khoddami MZ (2013) Nonlinear nonlocal vibration of embedded DWCNT conveying fluid using shell model. Phys B 410:188-196

34. Ghorbanpour AA, Amir S (2013) Electro-thermal vibration of visco-elastically coupled BNNT systems conveying fluid embedded on elastic foundation via strain gradient theory. Phys B 419:1-6

35. Eichler A, Moser J, Chaste J, Zdrojek M, Wilson-Rae RI, Bachtold A (2011) Nonlinear damping in mechanical resonators made from carbon nanotubes and grapheme. Nat Nanotechnol 6:339-342

36. Dodds HL, Runyan H (1965) Effects of high velocity fluid flow in the bending vibrations and static divergence of a simply supported pipe. National Aeronautical and Space Administration report, NASA TN, D-2870

37. Sobamowo MG (2017) Nonlinear thermal and flow induced vibration analysis of fluid-conveying carbon nanotube resting on Winkler and Pasternak foundations. Therm Sci Eng Prog 4:133-149

38. Liu F, Wagterveld RM, Gebben B, Otto MJ, Biesheuvel PM, Hamelers HV (2014) Carbon nanotube yarns as strong flexible conductive capacitive electrodes. Colloid Interface Sci Commun 3:9-12

39. Sobamowo MG, Yinusa AA (2018) Thermo-fluidic parameters effects on nonlinear vibration of fluid-conveying nanotube resting on elastic foundations using homotopy perturbation method. J Therm Eng 4(4):2211-2233

40. Coşkun SB, Atay MT, Öztürk B (2011) Transverse vibration analysis of Euler-Bernoulli beams using analytical approximate techniques. In: Ebrahimi $F$ (ed) Advances in vibration analysis research. ISBN: 978-953-307-209-8, InTech

41. Sobamowo MG, Yinusa AA (2017) Power series-after treatment technique for nonlinear cubic duffing and double-well duffing oscillators. J Comput Appl Mech 48(2):297-306

42. Eringen AC (1983) On differential equations of nonlocal elasticity and solutions of screw dislocation and surface waves. J Appl Phys 54(9):4703-4710 
43. Eringen AC (1972) Linear theory of nonlocal elasticity and dispersion of plane waves. Int J Eng Sci 10(5):425-435

44. Eringen AC, Edelen DGB (1972) On nonlocal elasticity. Int J Eng Sci 10(3):233-248

45. Eringen AC (2002) Nonlocal continuum field theories. Springer, New York

46. Sobamowo MG, Akinshiloa AT (2018) Double diffusive magnetohydrodynamic squeezing flow of nanofluid between two parallel disks with slip and temperature jump boundary conditions. Appl Comput Mech 11(2):167-182

47. Sobamowo MG, Adeleye OA (2018) Homotopy perturbation method for kinetic analysis of thermal inactivation of jack bean urease. Karbala Int J Mod Sci 4:187-199

Publisher's Note Springer Nature remains neutral with regard to jurisdictional claims in published maps and institutional affiliations. 